\title{
Peptide-based PET quantifies target engagement of PD-L1 therapeutics
}

\author{
Dhiraj Kumar, ${ }^{1}$ Ala Lisok, ${ }^{1}$ Elyes Dahmane, ${ }^{2}$ Matthew McCoy, ${ }^{3}$ Sagar Shelake, ${ }^{1}$ Samit Chatterjee, ${ }^{1}$ Viola Allaj, ${ }^{4}$ Polina Sysa-Shah, \\ Bryan Wharram, ${ }^{1}$ Wojciech G. Lesniak, ${ }^{1}$ Ellen Tully, ${ }^{5}$ Edward Gabrielson, ${ }^{5,6}$ Elizabeth M. Jaffee, ${ }^{5,6}$ John T. Poirier, ${ }^{4}$ \\ Charles M. Rudin, ${ }^{4}$ Jogarao V.S. Gobburu, ${ }^{2}$ Martin G. Pomper, ${ }^{1,6,7}$ and Sridhar Nimmagadda ${ }^{1,6,7,8}$
}

'The Russell H. Morgan Department of Radiology and Radiological Science, Johns Hopkins University School of Medicine, Baltimore, Maryland, USA. ${ }^{2}$ Center for Translational Medicine, Department of Pharmacy Practice and Science, University of Maryland School of Pharmacy, Baltimore, Maryland, USA. IInnovation Center for Biomedical Informatics, Georgetown University, Washington, DC, USA. ${ }^{4}$ Department of Medicine, Memorial Sloan Kettering Cancer Center, New York, New York, USA. ${ }^{5}$ Department of Pathology, Johns Hopkins University School of Medicine, Baltimore, Maryland, USA. ${ }^{6}$ The Sidney Kimmel Comprehensive Cancer Center and the Bloomberg-Kimmel Institute for Cancer Immunotherapy, Johns Hopkins University School of Medicine, Baltimore, Maryland, USA. PPharmacology and Molecular Sciences, Johns Hopkins University School of Medicine, Baltimore, Maryland, USA. ${ }^{8}$ Division of Clinical Pharmacology, Department of Medicine, Johns Hopkins University School of Medicine, Baltimore, Maryland, USA.

\begin{abstract}
Immune checkpoint therapies have shown tremendous promise in cancer therapy. However, tools to assess their target engagement, and hence the ability to predict their efficacy, have been lacking. Here, we show that target engagement and tumor-residence kinetics of antibody therapeutics targeting programmed death ligand-1 (PD-L1) can be quantified noninvasively. In computational docking studies, we observed that PD-L1-targeted monoclonal antibodies (atezolizumab, avelumab, and durvalumab) and a high-affinity PD-L1-binding peptide, WL12, have common interaction sites on PD-L1. Using the peptide radiotracer $\left[{ }^{64} \mathrm{Cu}\right] \mathrm{WL} 12$ in vivo, we employed positron emission tomography (PET) imaging and biodistribution studies in multiple xenograft models and demonstrated that variable PD-L1 expression and its saturation by atezolizumab, avelumab, and durvalumab can be quantified independently of biophysical properties and pharmacokinetics of antibodies. Next, we used [ $\left.{ }^{64} \mathrm{Cu}\right] \mathrm{WL} 12$ to evaluate the impact of time and dose on the unoccupied fraction of tumor PD-L1 during treatment. These quantitative measures enabled, by mathematical modeling, prediction of antibody doses needed to achieve therapeutically effective occupancy (defined as $>\mathbf{9 0 \%}$ ). Thus, we show that peptidebased PET is a promising tool for optimizing dose and therapeutic regimens employing PD-L1 checkpoint antibodies, and can be used for improving therapeutic efficacy.
\end{abstract}

\section{Introduction}

The success of cancer drug therapy depends on a number of factors within the tumor including drug access, completeness of drugtarget engagement, and targeting cancer cell heterogeneity. Target engagement in particular, which includes timing and extent of the drug-target interaction, is poorly understood for the majority of

Authorship note: $\mathrm{DK}$ and $\mathrm{AL}$ contributed equally to this work.

Conflict of interest: DK, SC, WCL, MCP, and SN are co-inventors on a pending US patent (PCT/US2017/068025) covering $\left[{ }^{64} \mathrm{Cu}\right] \mathrm{WL} 12$ and as such are entitled to a portion of any licensing fees and royalties generated by this technology. This arrangement has been reviewed and approved by Johns Hopkins University in accordance with its conflict-of-interest policies. DK, SC, WCL, SN, JTP, CMR, and MCP are inventors on patent applications not related to this work. CMR serves on the Scientific Advisory Board of Harpoon Therapeutics, and has consulted on oncology drug development for AbbVie, Amgen, Ascentage, BMS, Celgene, Daiichi Sankyo, Genentech, Loxo, and Pharmamar. Under a licensing agreement between EMJ, Aduro Biotech Inc., and Johns Hopkins University, the University is entitled to milestone payments and royalties on sales of certain cancer vaccine products. EMJ serves on the Scientific Advisory Board of Genocea, Adaptive Biotech, DragonFly, and CSTONE and received grants from Aduro Biotech, Amgen, BMS, Hertix, and Corvus.

License: Copyright 2019, American Society for Clinical Investigation.

Submitted: June 5, 2018; Accepted: November 13, 2018.

Reference information: / Clin Invest. 2019;129(2):616-630.

https://doi.org/10.1172/JCI122216. anticancer therapeutics (1-3). Existing technologies such as mass spectrometry (4), cellular thermal shift assay (5), or fluorescence anisotropy imaging (6) assist in the characterization of drug activity in cells and tissues. However, they have limited application in quantifying completeness of drug-target engagement within and across multiple lesions simultaneously, and in real time. Such real-time measurements are important to capture the dynamic changes in tumor target expression, which impacts success as in the case of immune checkpoint therapy (7-9). Noninvasive imaging technologies such as positron emission tomography (PET) provide a precise means to quantify drug-target engagement in all lesions concurrently and can be used to address some of those challenges (10). PET is used to quantify target engagement and enable dose-finding of some small-molecule therapeutics developed for central nervous system disorders, but only rarely for those developed for oncology and for biologics (11). Specifically, PET has not been used to define the drug-target engagement of immune checkpoint therapeutics. Given the complex tumor and tissue responses associated with immune checkpoint therapy, quantification of target expression and completeness of drugtarget engagement, in principle, could be integrated into treatment protocols to improve efficacy, avoid off-target effects, and enable enrollment of patients most likely to respond. 

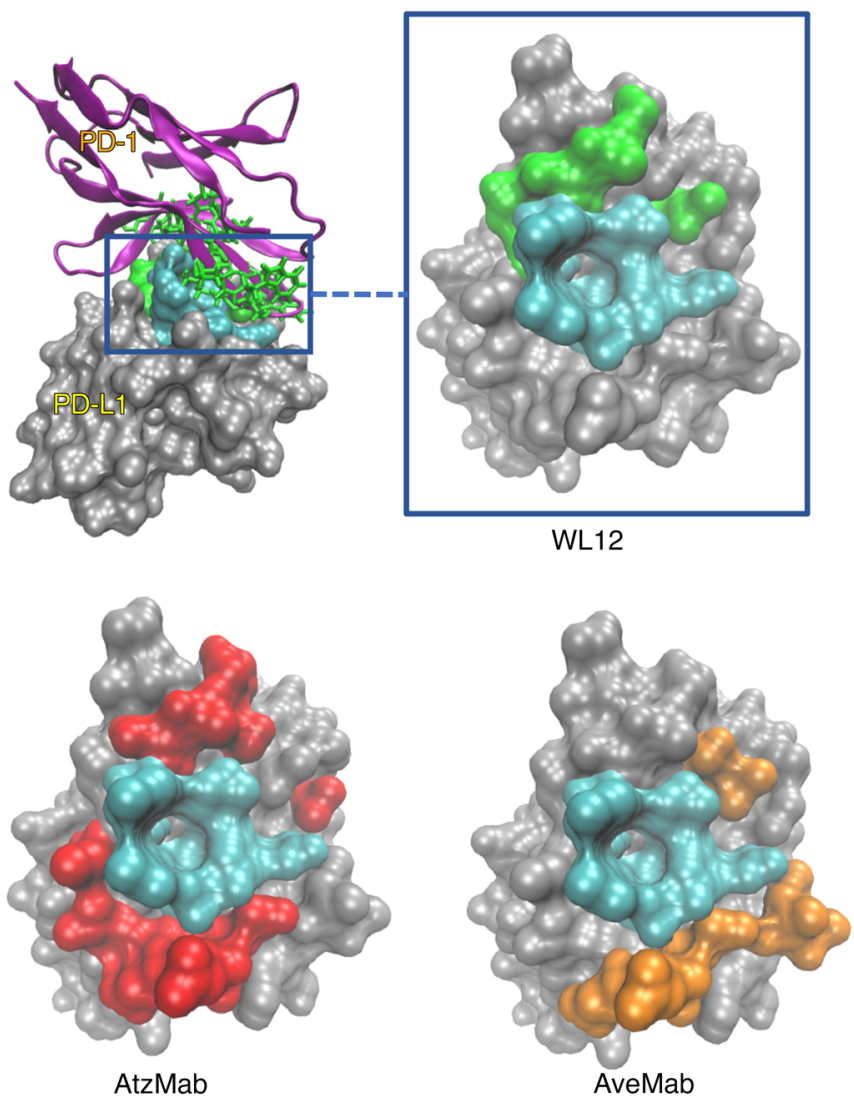

Nearly $70 \%$ of patients receiving immune checkpoint therapeutics do not respond (12). To date, most research into immune checkpoint resistance has focused on genetic and immunological factors such as tumor mutational burden, $\mathrm{T}$ cell clonality, programmed death ligand-1 (PD-L1, also known as B7-H1) expression, and CD8-positive cells in the tumors (13-15). However, due to lack of suitable technology, the relevance of dose to the degree and duration of target engagement within tumors remains unknown for immune checkpoint therapeutics. Understanding these dynamic properties would contribute to a more complete understanding of the targeted intervention and ensure optimum immune response.

PD-L1-targeted therapeutics form the backbone of immune checkpoint therapy today, and methods for detecting and quantifying PD-L1 are critical for guiding such therapy (16). Three PD-L1 therapeutic mAbs (atezolizumab [AtzMab], avelumab [AveMab], and durvalumab [DurMab]) have received FDA approval; however, the extent of PD-L1 engagement by these mAbs at the tumor remains unknown. That is because intratumoral concentrations of antibodies are influenced by multiple factors including dynamic changes in PD-L1 expression, parameters intrinsic to tumors such as vascularization and interstitial pressure, and extrinsic parameters related to their complex pharmacokinetics influenced by drug-target-complex turnover (17). Peripheral T cell-derived target-engagement data are often used for dose-finding of PD-L1 therapeutics, but it may not truly reflect target engagement at the tumor. For example, for the antibody BMS-936559, a PD-L1 occupancy of $64 \%-70 \%$ has been reported in $\mathrm{T}$ cells for doses ranging from 0.1 to $10 \mathrm{mg} / \mathrm{kg}$; however, occupancy and accessible PD-L1 levels in the tumor remain unknown (18). Radiolabeled antibod-

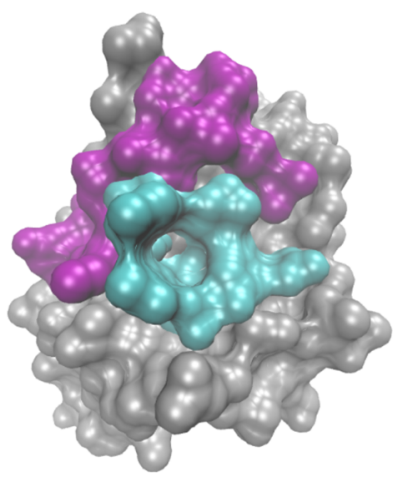

PD-1

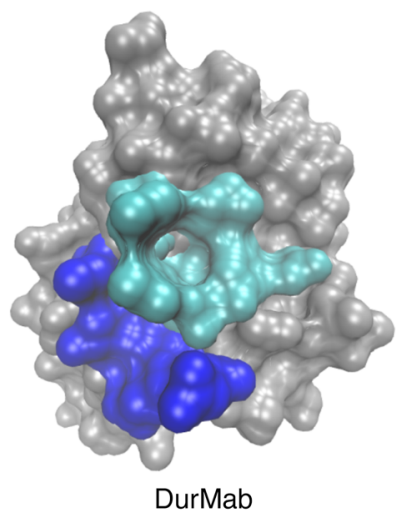

Figure 1. WL12 binding interface on PD-L1 overlaps with PD-1 and PD-L1 therapeutics. (A) WL12 binding mode to PD-L1 (green and cyan) overlaps those of PD-1 (purple and cyan), AtzMab (red and cyan), AveMab (orange and cyan), and DurMab (blue and cyan). Noninteracting residues are shown in gray. The variety of contacts region (cyan) illustrate the diverse binding mechanisms of different therapeutic antibodies. encompassing the shared binding

ies can provide information about tumor accumulation at a given dose but have not been used to calculate the extent of PD-L1-drug engagement in patients, in part due to their prolonged circulation time (19). Such findings underscore the need for quantitative measurement of PD-L1 target levels and their engagement by therapeutic antibodies at the tumor, accounting for antibody kinetics, at all identifiable lesions.

We previously developed a high-affinity PD-L1-specific peptide radiolabeled with ${ }^{64} \mathrm{Cu},\left[{ }^{64} \mathrm{Cu}\right] \mathrm{WL} 12$, which generates highcontrast images within 120 minutes of radiotracer administration (20). Herein we evaluate $\left[{ }^{64} \mathrm{Cu}\right] \mathrm{WL} 12$ PET to quantify dynamic changes in tumor PD-L1 expression and the potential for target engagement of FDA-approved PD-L1 therapeutics. Our results demonstrate that $\left[{ }^{64} \mathrm{Cu}\right] \mathrm{WL} 12$ quantified PD-L1 engagement at the tumor independently of antibody biophysical characteristics and in vivo kinetics. We hypothesize that $\left[{ }^{64} \mathrm{Cu}\right] \mathrm{WL} 12$ peptide-based PET will enable measurement of the PD-L1 engagement by any therapeutic antibody at the tumor to assist in improving outcomes in patients undergoing immunotherapy. These findings establish what we believe is a novel approach that can be generalized to study other therapeutic antibody interactions with their tumor target.

\section{Results}

Structural analysis of PD-L1 interactions with WL12 and PD-L1 $m A b s$. WL12 is a $14-$ amino acid circular peptide that binds PD-L1 with high affinity and disrupts interactions between PD-L1 and programmed death-1 receptor (PD-1) ( $\left.\mathrm{IC}_{50} 20 \mathrm{nM}\right)$. Our earlier molecular modeling analysis indicated an overlap in the interaction surface of PD-L1/WL12 and PD-L1/PD-1 complexes as the 
A

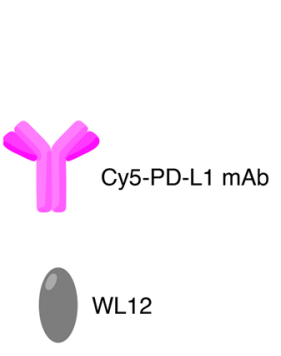

C

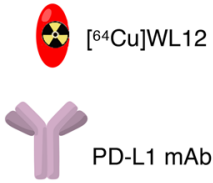

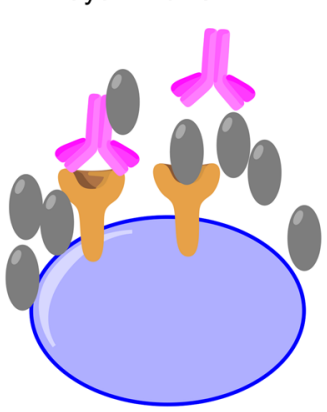

Cy5-mAb vs. WL12

$\left[{ }^{64} \mathrm{Cu}\right] \mathrm{WL} 12$ vs. mAb

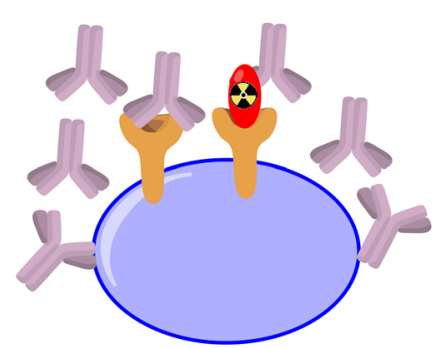

Figure 2. WL12 inhibits interaction between PD-1 and PD-L1 therapeutics in vitro. (A) Schematic representation of the assay. (B) WL12 inhibits Cy5-conjugated AtzMab, AveMab, and DurMab binding to PD-L1, as demonstrated through competitive inhibition and corresponding $\mathrm{IC}_{50}$ values. Mean fluorescence intensities (MFIs) were determined by flow cytometry. (C) Schematic representation of the assay. (D) [ $\left.{ }^{64} \mathrm{Cu}\right] \mathrm{WL} 12$ binding to PD-L1-positive HCC827, H226, $\mathrm{hPD}-\mathrm{L} 1$, and MDAMB231 cells is inhibited in the presence of $60 \mathrm{nM}$ AtzMab, AveMab, and DurMab, compared with PBS control. [ $\left.{ }^{64} \mathrm{Cu}\right]$ WL12 binding in PD-L1-negative CHO and SUM149 cells is also shown. ${ }^{* * *} P<0.0001$; NS, not significant, by 1 -way ANOVA and Dunnett's multiple comparisons test in $\mathbf{D}$.

B
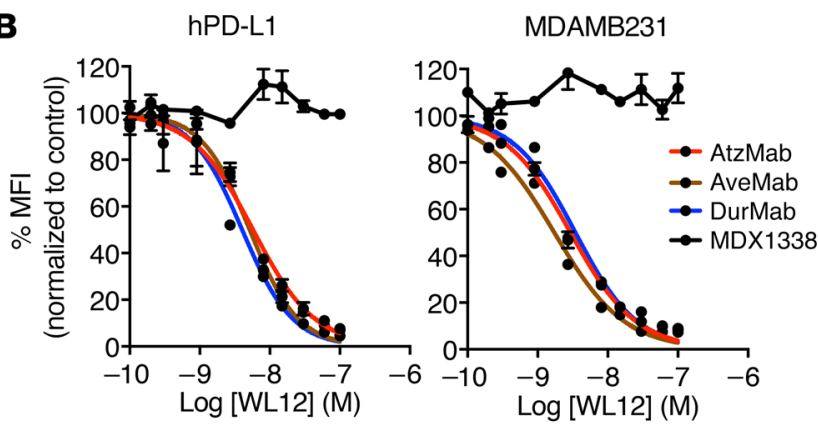

D
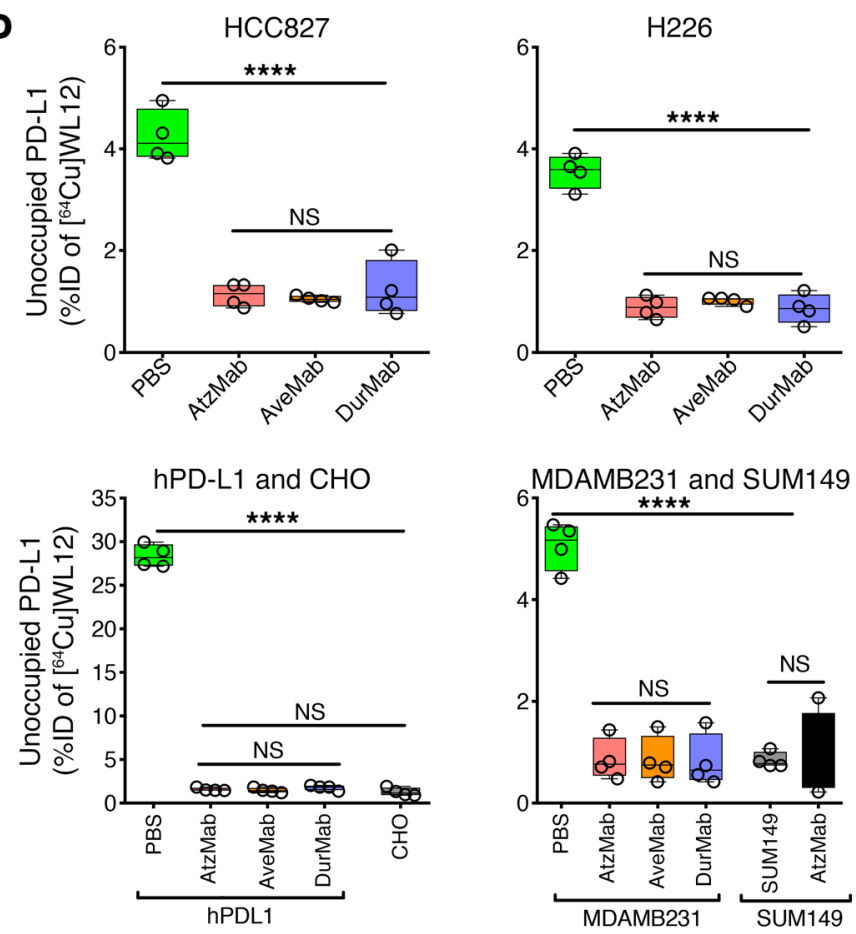

reason for the high affinity (20), which led us to hypothesize that the WL12 interaction surface on PD-L1 also overlaps with that of clinically available antibody therapeutics designed to inhibit PD-L1/PD-1 interactions. To test our hypothesis we compared the predicted binding conformations of WL12 with those of reported PD-L1 antibodies. The overlap of amino acid contacts between PD-L1 and PD-L1 antibodies, a PD-L1 nanobody (KNO35), as well as PD-1 and WL12, revealed a common binding domain composed of PD-L1 residues Y56, E58, A113, M115, and Y123. As shown in the visualization of the PD-L1 molecular surface (Figure 1 and Supplemental Figure 1; supplemental material available online with this article; https://doi.org/10.1172/JCI122216DS1), a singular overlapping region (cyan) forms a deep binding pocket, and acts as an anchor point for interactions with all of the agents investigated. The presence of a shared binding interface (cyan) is a strong indication that WL12 (green) competes with both the natural ligand (PD-1, purple) and antibodies. Further supporting our PD-L1/WL12 structural analysis, the shared binding interface has been observed with other macrocyclic peptide inhibitors targeted at disrupting PD-L1/PD-1 interactions (21). In terms of surface area, AtzMab (cyan and red) interacts with more of the PD-L1 sur- face than the other agents, with loops from the AtzMab generating molecular contacts with residues on all sides of the shared binding interface. Also, AtzMab overlaps with the interaction surfaces of PD-1 (cyan and purple), WL12 (cyan and green), AveMab (cyan and orange), and DurMab (cyan and blue). On the other hand, DurMab and AveMab show less overlap with the PD-1 interaction surface on PD-L1, further supporting the hypothesis that a shared binding interface pocket must be a core in the functional interaction for all the investigated agents. Those observations provide evidence that WL12 could be useful to detect and quantify antibody binding to the target.

In vitro validation of $P D-L 1$ interactions with WL12 and PD-L1 $m A b s$. To validate the observations from structural analysis, we prepared Cy5-labeled AtzMab, AveMab, and DurMab analogs through conjugation of the fluorescent $\mathrm{Cy} 5 \mathrm{~N}$-hydroxysuccinimide (NHS) ester to each mAb. Matrix-assisted laser desorption ionization time-of-flight (MALDI-TOF) mass spectrometric analyses of the purified conjugates showed an average of 1 to $2 \mathrm{Cy} 5$ molecules per mAb (Supplemental Figure 2A). All 3 Cy5-mAb conjugates detected variable levels of PD-L1 expression in cell lines, with minor differences in mean fluorescence intensities (MFIs), and 


\section{Table 1. WL12 inhibits Cy5-conjugated AtzMab, AveMab, and DurMab binding to PD-L1}

$\begin{array}{lccc} & \text { PD-L1 antibody } & \text { IC }_{50}[\mathbf{n M}] & \mathbf{9 5 \%} \mathbf{C l} \\ \text { hPDL1 } & \text { AtzMab } & 5.758 & 4.373 \text { to } 7.583 \\ & \text { AveMab } & 5.155 & 4.349 \text { to } 6.11 \\ \text { MDAMB231 } & \text { DurMab } & 3.453 & 2.974 \text { to } 4.008 \\ & \text { AtzMab } & 2.934 & 2.555 \text { to } 3.369 \\ & \text { AveMab } & 1.726 & 1.44 \text { to } 2.068 \\ & \text { DurMab } & 4.025 & 3.398 \text { to } 4.766\end{array}$

with variable endogenous expression (20). Accordingly, we selected multiple cell lines with high endogenous PD-L1 expression (HCC827, H226, MDAMB231, and hPD-L1). Cells were incubated with $\left[{ }^{64} \mathrm{Cu}\right] \mathrm{WL} 12$ at $4^{\circ} \mathrm{C}$ for 30 minutes, washed, and cell-bound activity was measured. We observed high expression-dependent uptake of $\left[{ }^{64} \mathrm{Cu}\right]$ WL12 in those cells and uptake in PD-L1-positive cells reflected variable levels of surface PD-L1 expression observed by flow cytometry (Supplemental Figure 2B) in the order hPD-L1 > MDAMB231 > HCC827 > H226. PD-L1 specificity of $\left[{ }^{64} \mathrm{Cu}\right] \mathrm{WL} 12$ uptake was low in PD-L1-negative cells (SUM149 and CHO, $P<0.0001$ ). We observed significant blockade of $\left[{ }^{64} \mathrm{Cu}\right] \mathrm{WL} 12$ uptake in all PD-L1positive cells treated with $60 \mathrm{nM}$ mAb compared with consistent with routinely used commercial PD-L1 detection antibodies (Supplemental Figure 2B). To assess the specificity of WL12 for inhibiting binding of antibodies to PD-L1, we performed competitive inhibition assays in $\mathrm{CHO}$ cells constitutively expressing PD-L1 (hPD-L1) and in MDAMB231 triple-negative breast cancer (TNBC) cells, which naturally express PD-L1 (22). We observed that WL12 dose-dependently inhibits Cy5-mAb binding to PD-L1, with $\mathrm{IC}_{50}$ values of 2 to $5 \mathrm{nM}$ (Figure 2, A and B, and Table 1). We also validated those observations in HCC827 and H226 non-small cell lung cancer (NSCLC) cells, each of which naturally expresses moderate levels of PD-L1. HCC827 and H226 cells were incubated with Cy5-AtzMab, -AveMab, and -DurMab in the presence of a single concentration of WL12 (5 nM) and analyzed by flow cytometry for unoccupied-PD-L1 levels. Indeed, a significant reduction $(P<0.001)$ in $\mathrm{Cy} 5-\mathrm{mAb}$ binding indicating low unoccupiedPD-L1 levels was detected in the presence of WL12. We obtained further confirmation of the specificity of the WL12/PD-L1 interaction through the absence of a change in bound fluorescence when MDX1338, a CXCR4-specific (control) antibody, was used. Data in multiple cell lines with variable PD-L1 expression levels established the capacity of WL12 to disrupt antibody-PD-L1 interactions (Supplemental Figure 2, C and D). We next performed a bioluminescence-based functional assay to demonstrate the ability and potency of WL12 peptide to inhibit PD-1/PD-L1 interactions compared to PD-L1 antibodies (Supplemental Figure 2E). An in vitro functional assay showed that WL12 peptide is 10-fold less potent than AtzMab in inhibiting PD-1/PD-L1 interactions and therefore unlikely to interfere with an ongoing antibody therapy.

Mutation in PD-L1 could impact binding of PD-L1 imaging agents and therapeutics. Analysis of cBioportal and CCLE databases indicated mutations in PD-L1 in a small percentage of tumors and cell lines (Supplemental Figure 2, F-H). Uptake studies of $\left[{ }^{64} \mathrm{Cu}\right]$ WL12 in HCC1569 cells with an M115T mutation that is at the interface of PD-L1/WL12 showed significant increase in $\left[{ }^{64} \mathrm{Cu}\right]$ WL12 uptake in IFN- $\gamma$-treated cells compared with untreated cells (Supplemental Figure 2I). These observations warrant further investigation into PD-L1 variants and their relevance to binding of PD-L1 inhibitors.

Next we sought to characterize the potential of the WL12 peptide-derived radiotracer to measure target engagement by PD-L1 antibodies. Previously, we showed that $\left[{ }^{64} \mathrm{Cu}\right] \mathrm{WL} 12$ bound PD-L1 with high selectivity in vitro in hPD-L1 and CHO cells. However, such selectivity has not been validated in human cancer cell lines
PBS-treated controls $(P<0.0001)$, indicating binding specificity of the radiotracer (Figure 2, $C$ and D). The in vitro results suggest that $\left[{ }^{64} \mathrm{Cu}\right]$ WL12 could be used to quantify PD-L1/antibody interactions and unoccupied-PD-L1 levels in tumors.

In vivo quantification of tumor PD-L1 engagement by AtzMab. NSCLC xenograft models were used to evaluate in vivo PD-L1 engagement by the therapeutic mAbs in a noninvasive manner. We selected those models because nearly $50 \%$ of NSCLCs are PD-L1 positive and PD-L1 IHC is used as a predictive biomarker in patients with NSCLC undergoing immune checkpoint therapy (23).

We used NOD SCID $\gamma$ (NSG) mice bearing H226 and HCC827 cell-derived xenografts that exhibit low and moderate PD-L1 expression, respectively, and treated them with a single dose of AtzMab $(20 \mathrm{mg} / \mathrm{kg})$ administered intravenously as a bolus, 24 hours before $\left[{ }^{64} \mathrm{Cu}\right]$ WL12 injection. PET images acquired 2 hours after $\left.{ }^{64} \mathrm{Cu}\right]$ WL12 injection and relatively 26 hours after AtzMab injection showed higher accumulation of $\left[{ }^{64} \mathrm{Cu}\right]$ WL12 in HCC827 tumors compared with $\mathrm{H} 226$. There was a clear reduction in the accumulation of radioactivity in tumors of AtzMab-treated mice, indicating reduced levels of available PD-L1 interaction sites compared with saline-treated controls (Figure 3, A and B). The PET imaging results were further confirmed by ex vivo measurements of biodistribution (Figure 3, D and E, and Supplemental Figure 3 , A and B), which showed significant reductions in $\left[{ }^{64} \mathrm{Cu}\right] \mathrm{WL} 12$ accumulation (as percentage of injected dose per gram of tissue $[\% \mathrm{ID} / \mathrm{g}])$ in AtzMab-treated mice compared with saline controls: $34 \%(P<0.0001)$ in $\mathrm{H} 226$ and $47 \%(P<0.001)$ in HCC 827 xenografts. Radiotracer uptake was corroborated by high PD-L1 staining intensity observed in HCC 827 xenografts compared with H227 tumors (Figure 3, C and F). The results demonstrate that $\left[{ }^{64} \mathrm{Cu}\right] \mathrm{WL} 12 \mathrm{can}$ be used to assess, qualitatively and quantitatively, in vivo targeting of PD-L1 at the tumor by AtzMab.

Expression of PD-L1 varies highly between and across lesions in a given patient and tumor type such that different drug concentrations may be needed to establish a similar extent of target engagement and to reduce immune-related toxicities. To measure the effect of a single dose of AtzMab on targeting different PD-L1 levels in tumors, we performed PET and biodistribution studies in tumors derived from the hPD-L1 cells, which have 4- to 10-fold higher PD-L1 expression than HCC827 and H226 cells (Supplemental Figure 2B). The mice bearing contralateral hPD-L1 and CHO tumors (hPD-L1/CHO) were treated with AtzMab (20 mg/kg for 24 hours) and changes in tumor uptake 

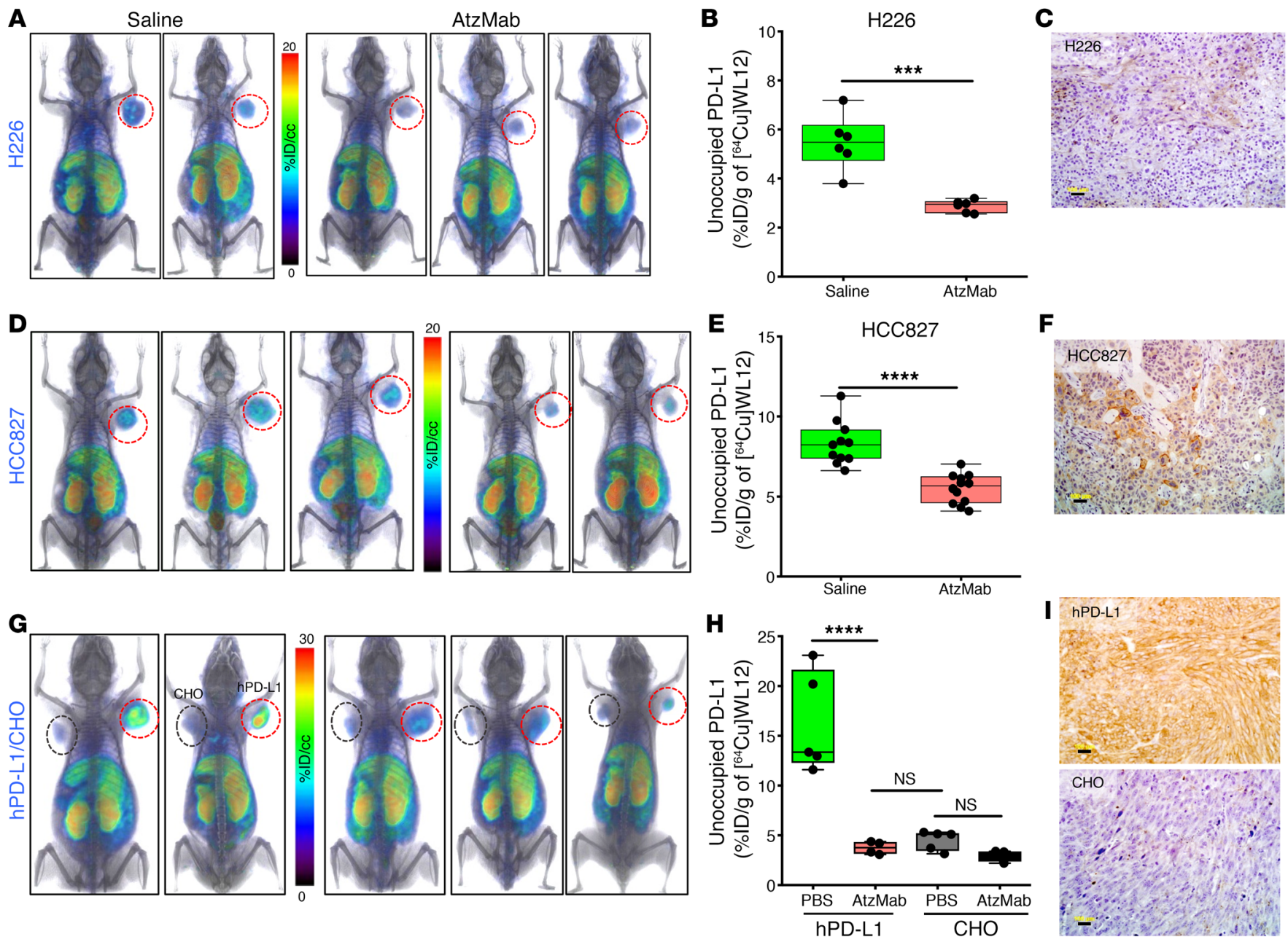

Figure 3. PD-L1 engagement by anti-PD-L1 mAbs is quantified at the tumor using $\left[{ }^{64} \mathrm{Cu}\right] \mathrm{WL} 12$ in xenografts with variable PD-L1 expression. (A-H) Reduced uptake of [ $\left.{ }^{64} \mathrm{Cu}\right] \mathrm{WL} 12$ in H226 (A and B), HCC827 (C and D), and contralateral hPD-L1 and PD-L1-negative CHO (hPD-L1/CHO) (G and H) xenografts in mice treated with $20 \mathrm{mg} / \mathrm{kg}$ AtzMab 24 hours prior to radiotracer injection, compared with saline-treated controls. Whole-body, volume-rendered [ $\left.{ }^{64} \mathrm{Cu}\right]$ WL12 PET-CT images (A, D, and $\mathbf{G})$ and ex vivo biodistribution (B, E, and $\mathbf{H})$ at 2 hours after $\left[{ }^{64} \mathrm{Cu}\right] \mathrm{WL} 12$ injection $(n=8-12 / g r o u p)$. (C, $\mathbf{F}$, and $\left.\mathbf{I}\right)$ IHC staining for PD-L1 is shown from the corresponding tumor. Scale bars: $100 \mu \mathrm{m}$. Box-and-whisker graphs showing minimum to maximum and all data points, with the horizontal line representing the median. ${ }^{* * *} P<0.0001 ;{ }^{* * *} P<0.001$; NS, not significant, by unpaired Student's $t$ test in $\mathbf{B}, \mathbf{E}$, and $\mathbf{H}$.

of $\left[{ }^{64} \mathrm{Cu}\right]$ WL12 were evaluated by PET. We observed high uptake of $\left[{ }^{64} \mathrm{Cu}\right]$ WL12 in hPD-L1 tumors (Figure 3G, right flank) compared with $\mathrm{CHO}$ tumors (left flank). Confirming AtzMab specific PD-L1 blockade, a significant reduction in $\left[{ }^{64} \mathrm{Cu}\right] \mathrm{WL} 12$ uptake was observed in hPD-L1 tumors in AtzMab-treated mice, compared with saline-treated controls (Figure 3G). Validating PET imaging data and revealing significant tumor PD-L1 engagement by AtzMab, biodistribution studies showed a 77\% reduction in $\left[{ }^{64} \mathrm{Cu}\right]$ WL12 uptake in the AtzMab-treated hPD-L1 tumors compared with saline controls (Figure $3 \mathrm{H}$ and Supplemental Figure $3 \mathrm{C})(P<$ 0.0001). In contrast, we observed only a low level of $\left[{ }^{[6} \mathrm{Cu}\right] \mathrm{WL} 12$ uptake in PD-L1-negative control CHO tumors. Also, $\left[{ }^{64} \mathrm{Cu}\right] \mathrm{WL} 12$ uptake in hPD-L1 tumors treated with AtzMab was not significantly different from that of $\mathrm{CHO}$ tumors, suggesting that treatment with a single $20 \mathrm{mg} / \mathrm{kg}$ dose of AtzMab led to near saturation of PD-L1 in those tumors. Those observations were confirmed by strong and weak immunoreactivity observed in hPD-L1 and CHO tumors, respectively (Figure 3I). Taken together, results from in vivo studies demonstrate that $\left[{ }^{64} \mathrm{Cu}\right] \mathrm{WL} 12 \mathrm{PET}$ can detect graded levels of PD-L1 expression in tumors, and can be used to quantify a wide range of PD-L1 engagement by a single $20 \mathrm{mg} / \mathrm{kg}$ dose of AtzMab in the tumors.

Quantifying dynamic changes in PD-L1 expression. PD-L1 is upregulated in response to various cytokines, importantly IFN- $\gamma$, which contributes to dynamic and spatiotemporal heterogeneity in PD-L1 expression $(24,25)$. However, those rapid changes have not been quantified in vivo in real time. We sought to evaluate $\left[{ }^{64} \mathrm{Cu}\right] \mathrm{WL} 12$ for its ability to quantify inducible PD-L1 expression within tumors in vivo (Figure 4), and to determine whether the blockade of such upregulated PD-L1 by AtzMab treatment could be monitored by $\left[{ }^{64} \mathrm{Cu}\right] \mathrm{WL} 12 \mathrm{PET}$.

We first generated an A549 NSCLC cell line with doxycycline-inducible PD-L1 expression (A549-iPDL1). A549 is a $\mathrm{KRAS}^{\mathrm{G} 12 \mathrm{~S}}$ human lung adenocarcinoma cell line that expresses low PD-L1 at baseline. It has been transduced with PD-L1 in the allin-one lentivirus pINDUCER20 vector (26), selected with G418, 


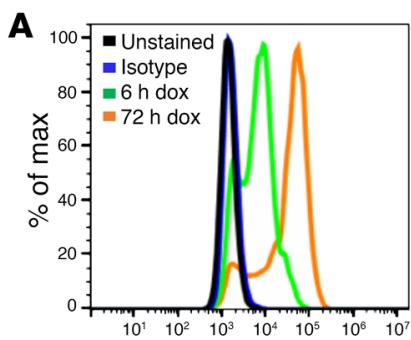

PE (anti-PD-L1)
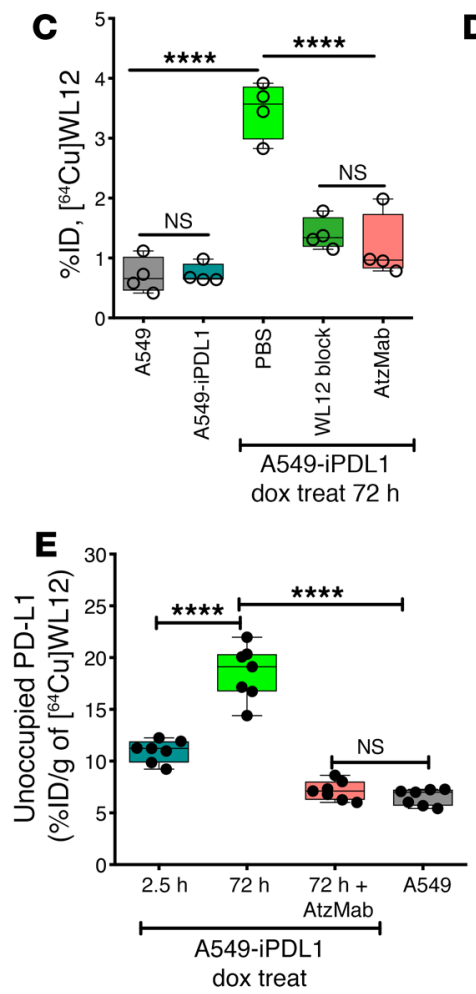

$\mathbf{F}$
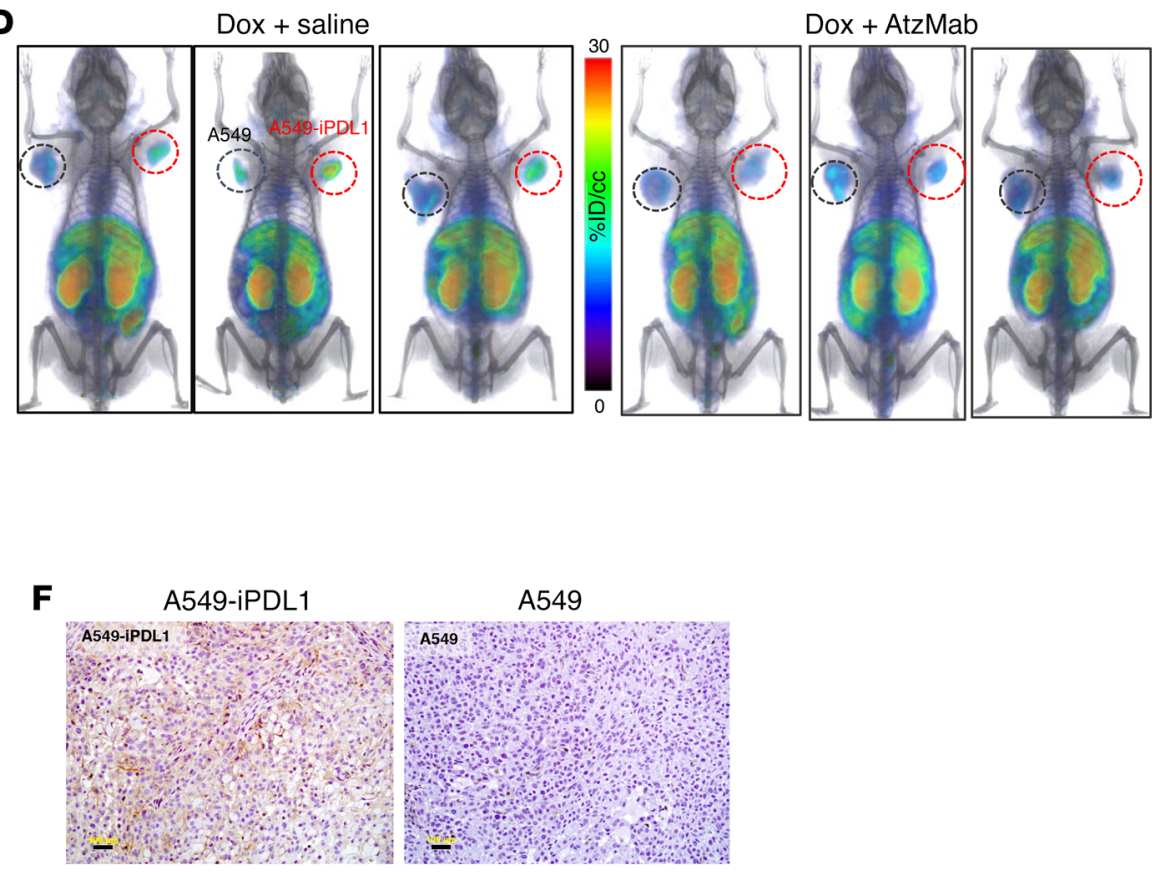

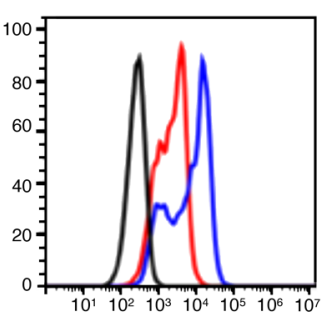

Cy5 (DurMab)
A549-iPDL1

Unstained

Cy5-Atz/Ave/Dur

WL12 blocking

Figure 4. Dynamic changes in tumor PD-L1 expression and its engagement by AtzMab detected using [ $\left.{ }^{64} \mathrm{Cu}\right]$ WL12. (A) Flow cytometry histogram showing increased PD-L1 cell surface expression in A549-iPDL1 cells treated with doxycycline for 6 hours and 72 hours. (B) WL12 inhibits (5 nM) binding of Cy5conjugated AtzMab, AveMab, and DurMab ( $2 \mathrm{nM}$ ) to A549-iPDL1 cells treated with doxycycline for 72 hours. (C) [ ${ }^{64} \mathrm{Cu}$ ]WL12 binding to A549-iPDL1 cells (72hour doxycycline) is significantly reduced in the presence of $60 \mathrm{nM}$ AtzMab, compared with controls. (D and E) [ ${ }^{64}$ Cu]WL12 uptake in A549-iPDL1 xenografts is significantly lower in mice receiving intravenous AtzMab 24 hours prior to radiotracer injection, compared with saline controls and similar to parent $A 549$ xenografts. Volume-rendered whole-body PET-CT images (D), and ex vivo quantification (E) at 2 hours after [ $\left.{ }^{64} \mathrm{Cu}\right] \mathrm{WL} 12$ injection ( $\left.n=10 / \mathrm{group}\right)$. (F) IHC staining for PD-L1 of the corresponding tumors. Scale bars: $100 \mu \mathrm{m}$. Box-and-whisker graphs showing minimum to maximum and all data points, with the horizontal line representing the median. ${ }^{* * *} P<0.0001$; NS, not significant, by 1-way ANOVA and Sidak's multiple comparisons test in $\mathbf{C}$ and $\mathbf{E}$.

confirmed for PD-L1 induction by flow cytometry (Figure 4A), and used for in vitro and in vivo studies. Validating the PD-L1 specificity of WL12, binding of Cy5-PD-L1 mAbs to doxycycline-treated A549-iPDL1 cells was blocked in the presence of WL12 (Figure 4B). Next, A549-iPDL1 cells were treated with doxycycline for 72 hours, incubated with radiotracer for 30 minutes, and cell-associated radioactivity was measured. Doxycycline-treated A549-iPDL1 cells showed increased surface PD-L1 expression, and exhibited a 5.5-fold higher uptake of $\left[{ }^{64} \mathrm{Cu}\right] \mathrm{WL} 12$, compared with untreated cells $(P<0.0001)$. We observed no significant differences in uptake between untreated A549-iPDL1 and parental A549 cells, confirming that $\left[{ }^{64} \mathrm{Cu}\right] \mathrm{WL} 12$ binding was specific to induced PD-L1 expression. Furthermore, $\left[{ }^{64} \mathrm{Cu}\right] \mathrm{WL} 12$ binding to doxycycline-treated A549-iPDL1 cells significantly decreased
$(65 \%, P<0.0001)$ in the presence of $60 \mathrm{nM}$ AtzMab, AveMab, and DurMab or nonradioactive WL12 (Figure 4C).

We next sought to validate those in vitro observations in vivo. NSG mice bearing A549-iPDL1 (Figure 4D, red circles) and A549 tumors (black circles) and treated with doxycycline for 72 hours were used. $\left[{ }^{64} \mathrm{Cu}\right]$ WL12 PET images and biodistribution studies of those mice showed high radioactivity uptake in A549-iPDL1 tumors. $\left[{ }^{64} \mathrm{Cu}\right] \mathrm{WL} 12$ in A549-iPDL1 tumors was $65 \%$ higher than in the A549 control tumors $(P>0.0001)$. Following AtzMab treatment, $\left[{ }^{64} \mathrm{Cu}\right] \mathrm{WL} 12$ uptake in A549-iPDL1 tumors was decreased more than $75 \%$, with \% ID/g values similar to control A549 tumors (Figure 4, D and E, and Supplemental Figure 4). IHC analysis of the tumors demonstrated an intense PD-L1 signal in A549-iPDL1 but not in A549 tumors, confirming imaging and biodistribution 
A

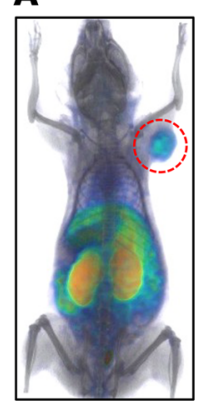

Saline

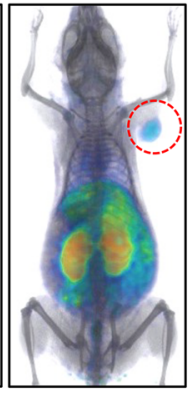

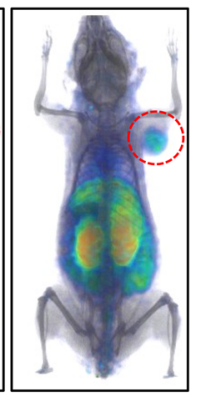

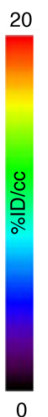

B

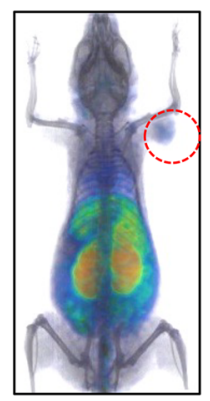

D

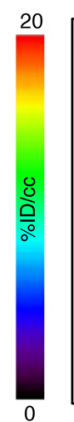

AveMab

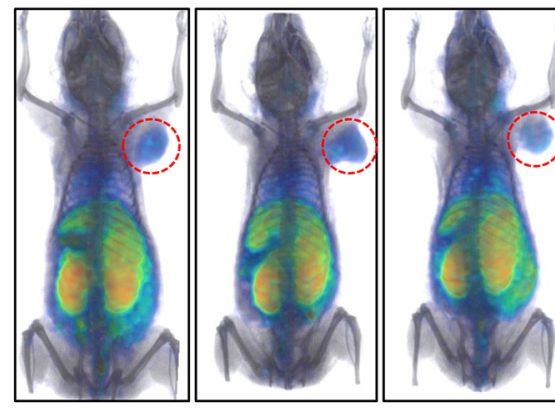

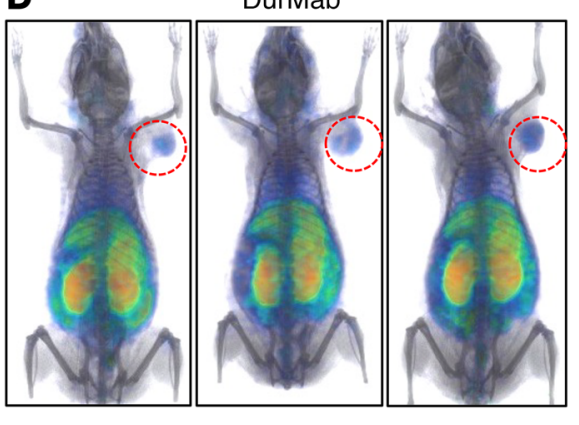

E

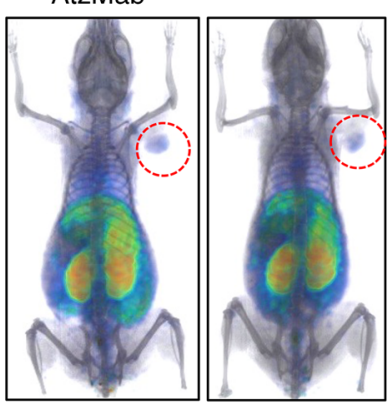

E

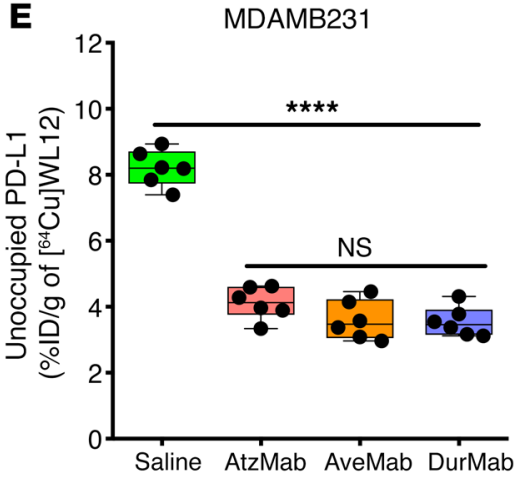

$\mathbf{F}$

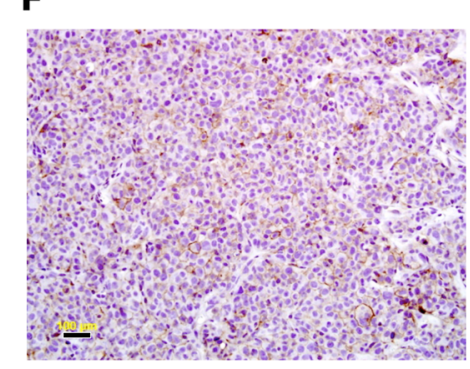

Figure 5. Tumor PD-L1 engagement by 3 distinct PD-L1 therapeutic antibodies quantified using [ $\left.{ }^{64} \mathrm{Cu}\right]$ WL12. (A-E) $\left[{ }^{64} \mathrm{Cu}\right]$ WL12 uptake in MDAMB231 xenografts is significantly reduced in mice receiving AtzMab $(20 \mathrm{mg} / \mathrm{kg})$, AveMab $(10 \mathrm{mg} / \mathrm{kg})$, or DurMab $(10 \mathrm{mg} / \mathrm{kg})$, 24 hours prior to radiotracer injection. Whole-body volume-rendered [ $\left.{ }^{64} \mathrm{Cu}\right]$ WL12 PET-CT images of saline- (A), AtzMab- (B), AveMab- (C), and DurMab-treated (D) mice, and ex vivo biodistribution (E) at 2 hours after $\left[{ }^{64} \mathrm{Cu}\right] \mathrm{WL} 12$ injection ( $n=6-9 /$ group). (F) IHC staining for PD-L1 in the corresponding tumor. Scale bars: $100 \mu \mathrm{m}$. Box-and-whisker graphs showing minimum to maximum and all data points, with the horizontal line representing the median. ${ }^{* * *} P<0.0001$; NS, not significant, by 1 -way ANOVA and Dunnett's multiple comparisons test in $\mathbf{E}$.

results (Figure $4 \mathrm{~F}$ ). Overall, the results demonstrate the potential of $\left[{ }^{64} \mathrm{Cu}\right]$ WL12 to detect dynamic changes in PD-L1 expression levels, and its blockade by AtzMab.

Quantifying target engagement in the tumors by different PD-L1 antibodies. We (22) and others (27-31) developed radiolabeled anti-PD-L1 antibodies and demonstrated their potential to assess PD-L1 expression in human tumor xenografts and syngeneic murine tumor models noninvasively. Observations made using radiolabeled antibodies, however, are highly specific to the antibody under investigation. In addition to the $3 \mathrm{FDA}$-approved PD-L1 mAbs, several others are in clinical development (32). A new approach is needed that (a) accounts for target engagement of PD-L1 antibodies, which (b) takes into account the properties of antibodies, and (c) is applicable to all PD-L1-targeted antibodies. Based on our in silico observations, we hypothesized that $\left[{ }^{64} \mathrm{Cu}\right]$ WL12 PET could be used to detect target engagement of any PD-L1 antibody that inhibits PD-L1/PD-1 interactions.

To test our hypothesis, we investigated the ability of $\left[{ }^{64} \mathrm{Cu}\right]$ WL12 PET to quantify PD-L1 engagement at the tumor by each of the 3 FDA-approved anti-PD-L1 mAbs. Moreover, those 3 mAbs

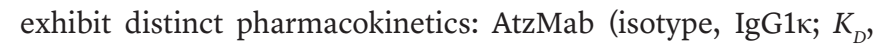
$0.4 \mathrm{nM} ; t_{1 / 2}, 27$ days), AveMab (IgG1 $\lambda, 0.7 \mathrm{nM}, 6.1$ days), and DurMab (IgG1 $\kappa, 0.022 \mathrm{nM}, 18$ days) (33). MDAMB231 tumor-bearing NSG mice were treated with a single dose of AtzMab $(20 \mathrm{mg} / \mathrm{kg})$, or AveMab $(10 \mathrm{mg} / \mathrm{kg})$, or DurMab (10 mg/kg) for 24 hours, and received $\left[{ }^{64} \mathrm{Cu}\right]$ WL12 for PET and biodistribution studies (Figure $5, \mathrm{~A}-\mathrm{D}) \cdot\left[{ }^{64} \mathrm{Cu}\right] \mathrm{WL} 12$ uptake was low in the tumors in all mAb-treat- ed mice, compared with saline controls, confirming low levels of unoccupied PD-L1 in the tumors due to PD-L1 engagement by the $\mathrm{mAbs}$ and radiotracer blockade. Ex vivo quantification of tumors validated those observations and demonstrated approximately $60 \%$ less uptake of $\left[{ }^{64} \mathrm{Cu}\right]$ WL12 in the tumors of mAb-treated mice $(P<0.0001)$ compared with saline controls (Figure $5 \mathrm{E}$ and Supplemental Figure 5). We have not observed significant differences in tumor PD-L1 blockade by different mAbs at the investigated dose and time, suggesting that all the mAbs demonstrate robust PD-L1 blockade at the tumor at early time points. IHC of saline controls demonstrated moderate to high PD-L1 intensity, placing these xenografts between highly PD-L1-positive hPD-L1 and weakly positive HCC827 and H226 tumors (Figure 5F). PD-L1 antibody treatment resulted in increased PD-L1 expression as an adaptive immune response to therapy in responders (34). Treatment of cell lines with PD-L1 mAbs had no effect on PD-L1 protein expression in the cell lines tested (Supplemental Figure 5B). The results demonstrate that tumor PD-L1 engagement by PD-L1 antibodies can be quantified by $\left[{ }^{64} \mathrm{Cu}\right] \mathrm{WL} 12$ PET and independently of the plasma and tissue kinetics exhibited by a given PD-L1 antibody. Importantly, this approach could provide a method to evaluate biosimilar antibodies as they are developed to facilitate the time to approval and reduce clinical testing expense.

Effect of dose and time on PD-L1 occupancy at the tumor. Antibody kinetics at the tumor are complex and governed by both tumor-intrinsic and -extrinsic parameters (17). We (22) and others (29) recently discovered that factors other than PD-L1 expres- 
A

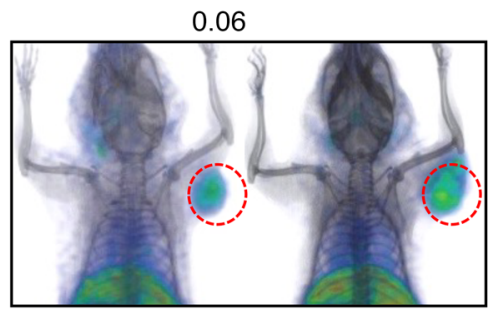

C

$24 \mathrm{~h}$
AtzMab dose $(\mathrm{mg} / \mathrm{kg})$, 24-h treatment 0.6

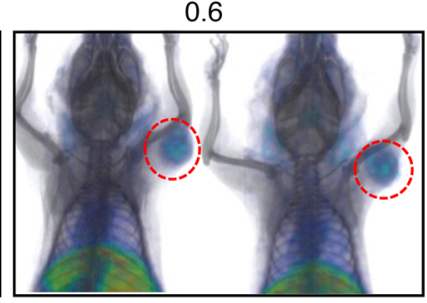

$0.6 \mathrm{mg} / \mathrm{kg}$ AtzMab dose treatment

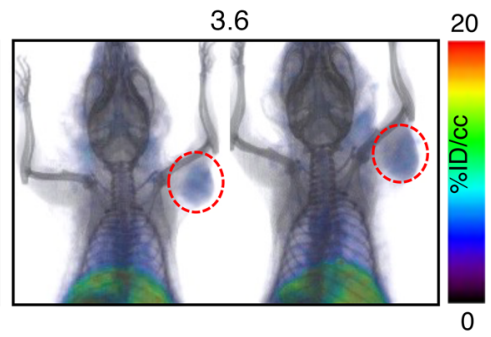

$120 \mathrm{~h}$
B

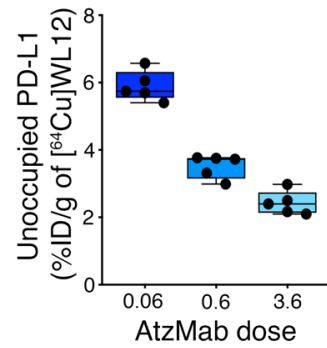

$(\mathrm{mg} / \mathrm{kg})$
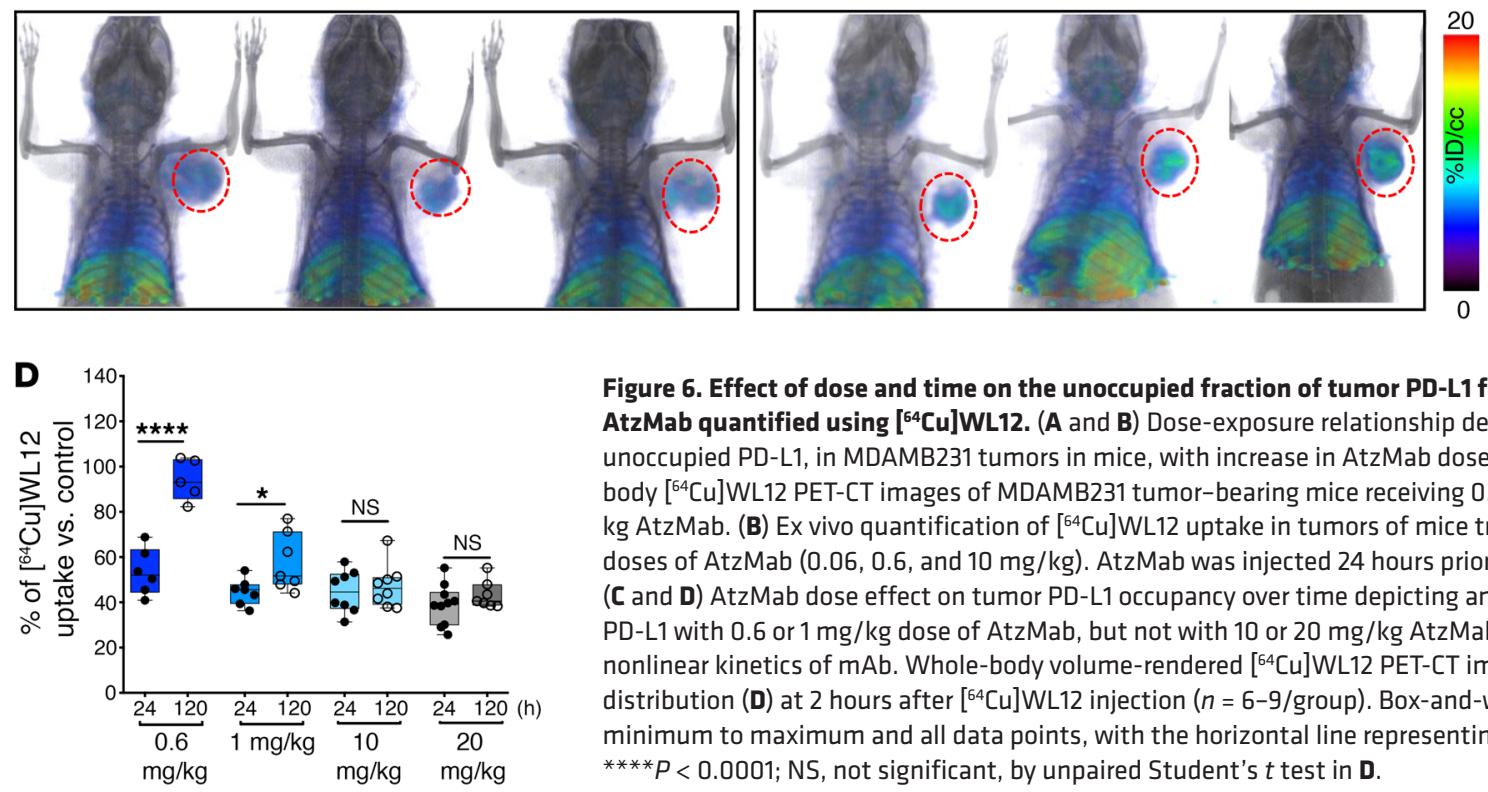

Figure 6. Effect of dose and time on the unoccupied fraction of tumor PD-L1 following treatment with

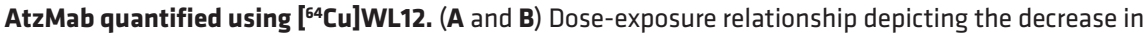
unoccupied PD-L1, in MDAMB231 tumors in mice, with increase in AtzMab dose $(\mathrm{mg} / \mathrm{kg})$. (A) Wholebody $\left[{ }^{64} \mathrm{Cu}\right] \mathrm{WL} 12 \mathrm{PET}-\mathrm{CT}$ images of MDAMB231 tumor-bearing mice receiving $0.06,0.6$, and $3.2 \mathrm{mg} /$ $\mathrm{kg}$ AtzMab. (B) Ex vivo quantification of [ $\left.{ }^{[64} \mathrm{Cu}\right] \mathrm{WL} 12$ uptake in tumors of mice treated with escalating doses of AtzMab $(0.06,0.6$, and $10 \mathrm{mg} / \mathrm{kg})$. AtzMab was injected 24 hours prior to radiotracer injection. (C and D) AtzMab dose effect on tumor PD-L1 occupancy over time depicting an increase in unoccupied PD-L1 with 0.6 or $1 \mathrm{mg} / \mathrm{kg}$ dose of AtzMab, but not with 10 or $20 \mathrm{mg} / \mathrm{kg}$ AtzMab dose, recapitulating the nonlinear kinetics of mAb. Whole-body volume-rendered $\left[{ }^{[64} \mathrm{Cu}\right] \mathrm{WL} 12$ PET-CT images (C) and ex vivo biodistribution (D) at 2 hours after $\left[{ }^{64} \mathrm{Cu}\right] \mathrm{WL} 12$ injection ( $n=6-9 /$ group). Box-and-whisker graphs showing minimum to maximum and all data points, with the horizontal line representing the median. ${ }^{*} P<0.05$, ${ }^{* * * *} P<0.0001$; NS, not significant, by unpaired Student's $t$ test in $\mathbf{D}$.

sion can reduce accumulation of the PD-L1-targeted therapeutic AtzMab and its mouse chimera (PRO304397) within NSCLC, TNBC, and colon tumors. Furthermore, experiments in mice showed that at doses less than $1 \mathrm{mg} / \mathrm{kg}$, the radioactivity measured from the systemically injected, radiolabeled PRO304397 was primarily associated with tumor vasculature, and showed minimal diffusion into tumor parenchyma in PD-L1-expressing tumor models (29). Those findings may be attributable to factors such as elevated intratumoral interstitial pressure, which impedes accumulation of antibodies in tumors, and estimated to be approximately $10 \%-30 \%$ of plasma levels (35-37). Such effects might also impede the access of large PD-L1-directed agents to targeted tumor cells and immune infiltrates. Occupancy measurements of PD-L1 therapeutics have been restricted to assessments made using peripheral blood mononuclear cells (PBMCs), in order to determine the dissociation constants of the drugs. However, PD-L1 occupancy measurements at the tumor have not yet been reported, and could provide a direct readout of therapeutic activity in the tumor.

To evaluate the effect of anti-PD-L1 therapeutic dose on tumor PD-L1 occupancy at the tumor, mice bearing MDAMB231 tumors were treated with single doses of AtzMab (0.06, 0.6, and $3.6 \mathrm{mg} / \mathrm{kg}$ body weight). Imaging and biodistribution studies were performed 2 hours after the injection of $\left[{ }^{64} \mathrm{Cu}\right] \mathrm{WL} 12$ and relatively 26 hours after AtzMab administration. PET images of mice that received $0.06 \mathrm{mg} / \mathrm{kg}$ showed high $\left[{ }^{64} \mathrm{Cu}\right] \mathrm{WL} 12$ uptake, indicating low PD-L1 occupancy by AtzMab at the tumor (Figure 6, A and B). At the higher doses $(0.6$ and $3.6 \mathrm{mg} / \mathrm{kg})$, there was a proportional decrease in $\left[{ }^{64} \mathrm{Cu}\right] \mathrm{WL} 12$ uptake in the tumors, indicating proportionally higher target engagement at the tumor by AtzMab. Corresponding biodistribution studies also demonstrated reduced unoccupied-PD-L1 levels in the tumors with increased dose, confirming that antibody dose-related changes in PD-L1 occupancy could be measured using $\left[{ }^{64} \mathrm{Cu}\right] \mathrm{WL} 12$.

Next, we sought to investigate the temporal changes in PD-L1 occupancy for a given AtzMab dose. Membrane-associated antigens such as PD-L1 can enhance antibody clearance through target-mediated endocytosis. Additionally, development of anti-therapeutic antibodies could significantly impact antibody serum and tumor kinetics (38). In support of those factors at play, earlier studies showed that AtzMab followed nonlinear kinetics below $0.6-1 \mathrm{mg} / \mathrm{kg}$ and linear kinetics above $1 \mathrm{mg} / \mathrm{kg}$, and a tendency toward reduced serum concentration in patients that developed anti-therapeutic antibodies (39). All of those factors will influence unoccupied-PD-L1 levels and tumor exposure and response to therapy. The tools to measure the effect of those factors on the tumor exposure to the anti-PD-L1 therapeutics in real time have not been described.

To investigate the ability of $\left[{ }^{64} \mathrm{Cu}\right]$ WL12 PET to detect temporal changes in antibody kinetics at the tumor, MDAMB231 


\section{Table 2. Pharmacodynamic model parameters describing the relationship between anti-PD-L1 antibody dose and the decrease in unoccupied PD-L1 measured at the tumor site}

\begin{tabular}{lc}
$\begin{array}{l}\text { Pharmacodynamic model parameters, } \\
\text { units }\end{array}$ & $\begin{array}{c}\text { Mean parameter value } \\
\text { (\% relative standard error) }\end{array}$ \\
\hline Baseline $_{\text {unoccupied PD-Ll, }} \% \mathrm{ID} / \mathrm{g}$ & $5.91(1 \%)$ \\
$\mathrm{I}_{\max }$ & $0.57(3 \%)$ \\
$\mathrm{ID}_{50}, \mathrm{mg} / \mathrm{kg}$ & $0.43(10 \%)$ \\
Hill coefficient & $3.12(19 \%)$ \\
Residual error, \%ID/g & $0.44(14 \%)$
\end{tabular}

tumor-bearing NSG mice were injected with AtzMab doses that were described to be associated with nonlinear $(0.6$ and $1 \mathrm{mg} /$ $\mathrm{kg}$ ) and linear kinetics (10 and $20 \mathrm{mg} / \mathrm{kg}$ ), and PET imaging and biodistribution studies were performed at 24 and 120 hours. At 24 hours, there was a significant reduction in $\left[{ }^{64} \mathrm{Cu}\right]$ WL12 uptake, also reflected in tumor \% ID/g values, in all 4 dose groups, compared with saline-treated controls (Figure 6, C and D). At 120 hours, there was a significant increase in $\left[{ }^{64} \mathrm{Cu}\right] \mathrm{WL} 12$ uptake in the 0.6 and 1 $\mathrm{mg} / \mathrm{kg}$ dose groups compared with 24 hours. At 120 hours, $\left[{ }^{64} \mathrm{Cu}\right]$ WL12 uptake in the tumor was similar in $0.6 \mathrm{mg} / \mathrm{kg}$-treated and saline control groups, suggesting the more rapid elimination of the drug from the tumor at the lowest dose. At the higher doses, however, $\left[{ }^{64} \mathrm{Cu}\right]$ WL12 uptake was significantly different from the saline control group, suggesting a slower elimination of the drug from the tumor, due to the saturable elimination or nonlinear pharmacokinetics of the drug at the higher doses. The temporal difference in $\left[{ }^{64} \mathrm{Cu}\right]$ WL12 uptake was not significant in the 10 or $20 \mathrm{mg} / \mathrm{kg}$ treatment groups, reflecting the linear pharmacokinetics or constant clearance of AtzMab at those doses. Collectively, the results show that in a mouse model both dose- and time-dependent changes in PD-L1 engagement can be quantitatively evaluated at the tumor.

Modeling dose-receptor occupancy of PD-L1 antibodies at the tumor. Pharmacokinetic-pharmacodynamic (PK/PD) modeling and simulation, linking the $\mathrm{PK}$ of the $\mathrm{mAb}$ to its receptor occupancy and tumor response, are routinely used as a modern approach for guiding starting dose selection from preclinical data, for firstin-human clinical trials, and for dose-range selection, in the later efficacy/safety clinical trials. Linking the receptor occupancy to the PK/PD model provides a mechanistic understanding of the antibody dose/concentration and tumor response relationship, and is valuable for efficient drug development and optimal dose selection $(17,40)$. PD-L1 expression in the tumors can be constitutive or induced, and its cell-surface stability could be modulated by oncogenic signaling and increased expression of positive regulators (7-9) that are difficult to account for based solely on in vitro assays. Those PK/PD mathematical models could be further refined using measurements of PD-L1 occupancy made at the tumor that encapsulate all the factors regulating PD-L1 levels and account for complex PD-L1 antibody kinetics.

To demonstrate the use of receptor occupancy measurements made at the tumor to define target-engagement characteristics and for dose finding, we measured unoccupied-PD-L1 levels in the tumors following AtzMab treatment. Mice bearing MDAMB231 tumors were injected with escalating doses of AtzMab from 0.009 to $24 \mathrm{mg} / \mathrm{kg}$ body weight. Twenty-four hours after AtzMab injections, biodistribution studies were performed 2 hours after the injection of $\left[{ }^{64} \mathrm{Cu}\right] \mathrm{WL} 12$. Because of the large number of groups involved, we relied on ex vivo biodistribution studies. A developed inhibitory sigmoidal $\mathrm{E}_{\max }$ model appropriately fitted and described the relationship between the dose of the AtzMab used in our experiment and the decrease in unoccupied PD-L1 at the tumor (Figure 7, A and B, and Supplemental Figure 6), detected using $\left[{ }^{64} \mathrm{Cu}\right] \mathrm{WL} 12$ and expressed as \% ID/g. The dose of the AtzMab responsible for $50 \%$ of the maximum PD-L1 engagement in the tumor $\left(\mathrm{ID}_{50}\right)$ was estimated to be $0.43 \mathrm{mg} / \mathrm{kg}$ (Table 2). The $\mathrm{ID}_{90}$ and $\mathrm{ID}_{96}$, responsible for $90 \%$ and $96 \%$ of the maximum fractional decrease in unoccupied PD-L1 from baseline $\left(\mathrm{I}_{\max }\right)$, corresponded to $0.87 \mathrm{mg} /$ $\mathrm{kg}$ and $1.19 \mathrm{mg} / \mathrm{kg}$, respectively. These dose levels are comparable to the dose of $1 \mathrm{mg} / \mathrm{kg}$ reported by Deng et al. for the chimeric anti-PD-L1 antibody PRO304397 and responsible for maximum PD-L1 saturation over 24 hours in BALB/c mice (29). Assuming a similar average volume of distribution at steady state $\left(\mathrm{V}_{\mathrm{ss}}\right)$ of 50 $\mathrm{ml} / \mathrm{kg}$ for the anti-PD-L1 and the chimeric anti-PD-L1 antibody PRO304397 (29), the expected average plasma concentrations resulting from $\mathrm{ID}_{50}, \mathrm{ID}_{90}$, and $\mathrm{ID}_{96}$ were tentatively estimated to be $59 \mathrm{nM}(8.6 \mu \mathrm{g} / \mathrm{ml}), 120 \mathrm{nM}(17.4 \mu \mathrm{g} / \mathrm{ml})$, and $164 \mathrm{nM}(23.8 \mu \mathrm{g} / \mathrm{ml})$, respectively. These results indicate the potential of using measurements made at the tumor, which reflect true target occupancy, for dose selection and optimization of future PD-L1 therapeutics.

\section{Discussion}

We have demonstrated the ability to measure PD-L1 occupancy using peptide-based PET according to a method that is agnostic to the choice of antibody. We were able to noninvasively quantify target engagement and pharmacokinetics of the PD-L1 mAbs at the tumor. The real-time measurements described encapsulate (a) the complex serum and tumor kinetics (or fate) of antibodies at the tumor site, (b) tumor-intrinsic parameters such as high interstitial pressure and poor vascularity that impede antibody accumulation, and (c) genetic and epigenetic mechanisms that regulate PD-L1 expression levels and turnover (Figure 7C). We further demonstrated the translational utility of our approach, by combining our experimentally derived, unoccupied-PD-L1 levels in the tumors with reported preclinical pharmacokinetics of AtzMab, to find a dose to achieve minimum efficacious occupancy levels at the tumor, as previously suggested (29).

Biologics such as antibodies pose unique challenges in predicting in vivo target engagement based on in vitro binding affinity (17). For small molecules, in vitro binding affinity measurements and target occupancy studies are routinely used for dose selection in central nervous system diseases and are predictive of pharmacological response (41). However, properties intrinsic to antibodies, such as valence, shape, size, and isoelectric point, make the pharmacokinetics of antibodies more complex and difficult to predict. Also, several parameters intrinsic to the tumor, such as antigen density and turnover, tumor burden, and tumor perfusion, limit intratumoral penetration of antibodies. Tumor and plasma concentrations of antibodies are further influenced by factors extrinsic to tumors such as dose, patient variability, cachexia, and development of anti-therapeutic antibodies (42). 
A

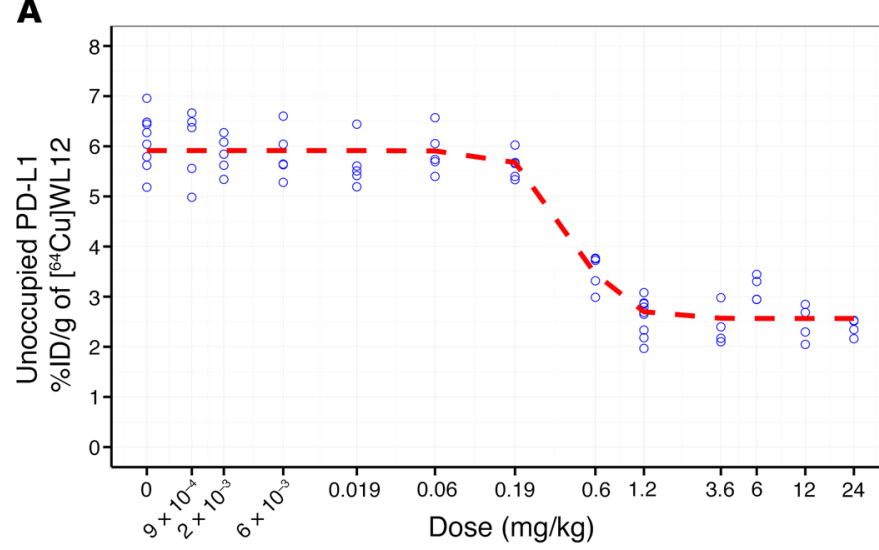

C

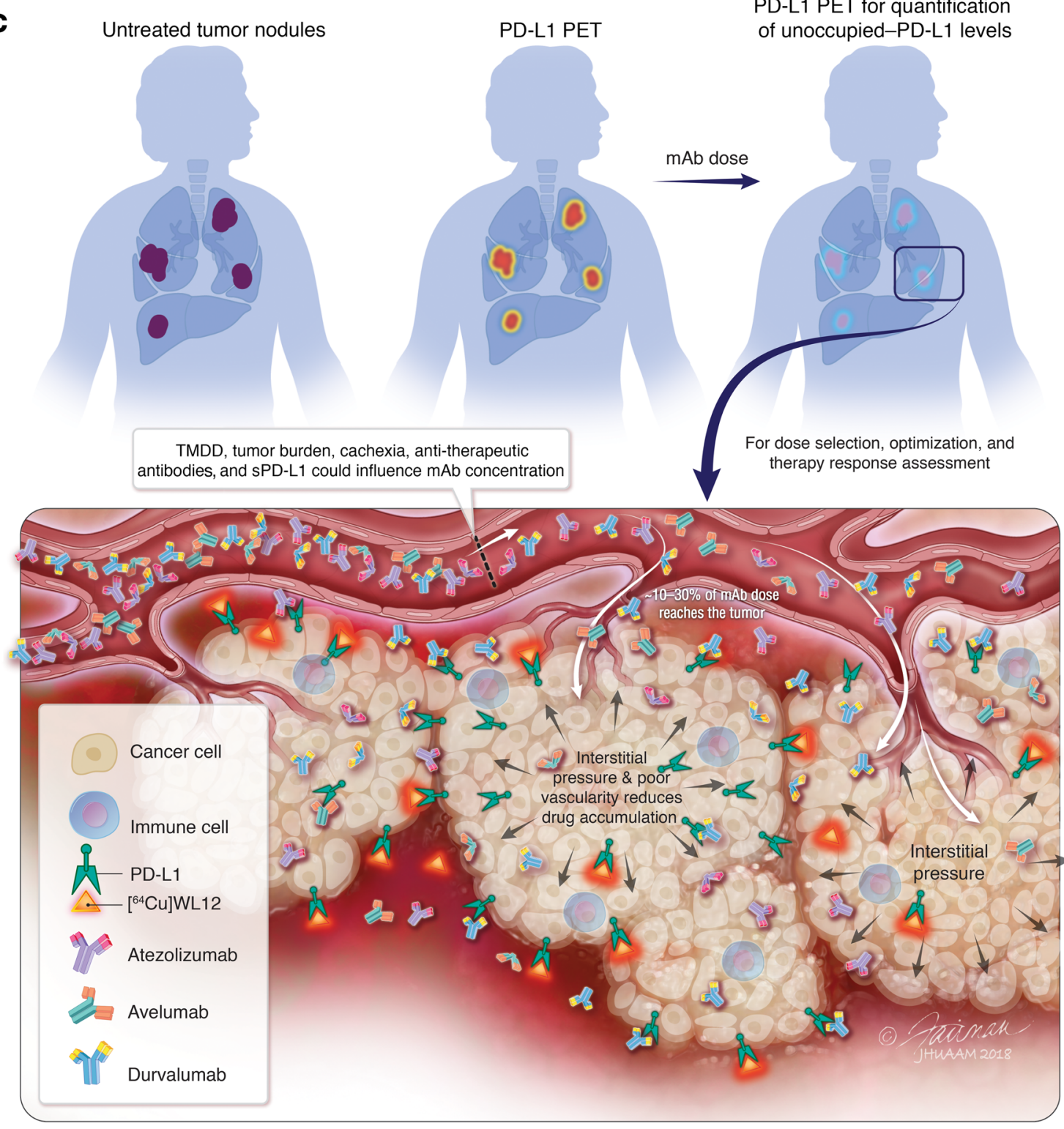

B

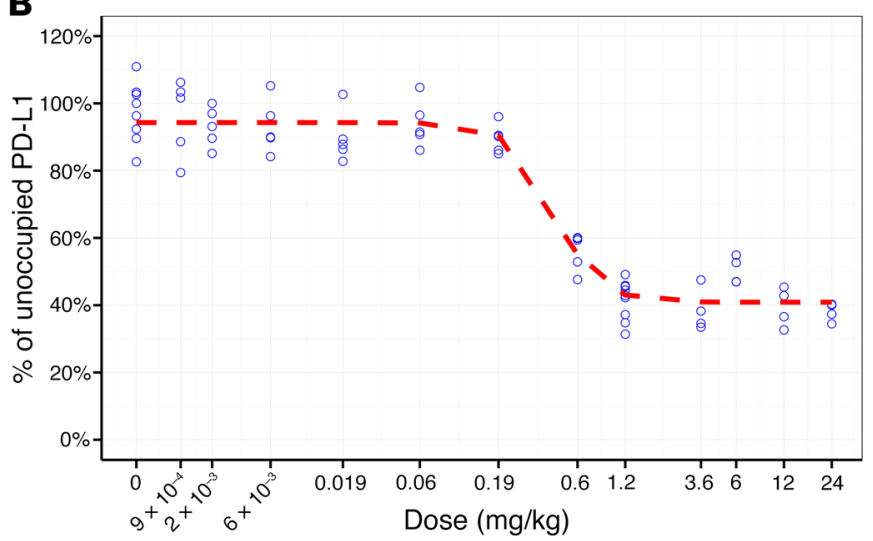

PD-L1 PET for quantification of unoccupied-PD-L1 levels

Figure 7. Effect of dose and time on tumor PD-L1 occupancy by AtzMab quantified using [ $\left.{ }^{64} \mathbf{C u}\right]$ WL12. (A and B) Dose-exposure relationship depicting the decrease in unoccupied PD-L1, in MDAMB231 tumors in mice, with increase in AtzMab dose ( $\mathrm{mg} / \mathrm{kg})$. Ex vivo quantification of [ $\left.{ }^{64} \mathrm{Cu}\right] \mathrm{WL} 12$ uptake at 2 hours in tumors of mice treated with escalating doses of AtzMab ( 0.0009 to $24 \mathrm{mg} / \mathrm{kg}$ ) ( $n=3-9 / \mathrm{group})$. AtzMab was injected 24 hours prior to radiotracer injection (A). Percentage of unoccupied PD-L1 was calculated relative to the median unoccupied PD-L1 measured at $0 \mathrm{mg} / \mathrm{kg}$ (B). Blue open dots: measured unoccupied PD-L1 for each dose level in mice. Red dashed line: mean model-predicted dose-response relationship. (C) Schematic of PD-L1 PET for PD-L1 therapeutic development and evaluation. SPD-L1, soluble PD-L1; TMDD, target-mediated drug disposition. 
All of these factors affect tissue exposure of antibodies and ultimately efficacy. For example, pidilizumab, developed with investments of over $\$ 200$ million, was later discovered not to bind PD-1, its intended target (43). Our approach accounts for target engagement of PD-L1 antibodies at the site of action and is applicable to all PD-L1 antibodies that inhibit PD-L1/PD-1 interactions.

The methods described here can be extended to evaluate the changes in unoccupied PD-L1 in all lesions in a patient concurrently, and used to further guide therapeutic decisions. Although IHCbased clinical tests are used to assess PD-L1 expression in tumors $(34,44,45)$, PD-L1 IHC takes into account only a small fraction $(0.1 \%)$ of a single lesion. Such an approach has significant limitations, because PD-L1 expression in the tumor microenvironment is spatially and temporally heterogeneous $(46,47)$. Also, tissue samples acquired by biopsy for testing are typically very limited, and may be needed for molecular profiling to identify targetable oncogenic mutations in other pathways that confer sensitivity or resistance to existing therapies (48). As a result of that heterogeneity, the sensitivity of a single small tissue sample can be low for detecting PD-L1 expression at scoring thresholds commonly used in clinical practice (49). Furthermore, immune therapy responses are delayed, complex, and abscopal in nature and it is impractical to perform multiple biopsies in multiple lesions for reliable representation of PD-L1 expression (50). Those issues are compounded in patients with metastatic disease, a population in which immune checkpoint therapeutics are extensively investigated.

New regulators of PD-L1 are emerging that control cell surface PD-L1 levels. The CKLF-like MARVEL transmembrane domaincontaining proteins 4 and 6 (CMTM 4/6) $(7,8)$ and oncogenic proteins such as mutant RAS have been shown to increase cell surface PD-L1 protein lifetime or PD-L1 expression (9). Such PD-L1 dynamics are difficult to capture by IHC-based assessments and contribute to our limited success in advancing immunotherapies. Our studies with $\left[{ }^{64} \mathrm{Cu}\right] \mathrm{WL} 12$ demonstrate that variable and dynamic changes in PD-L1 expression could be quantified during therapy and within the standard clinical work flow, yielding important clinical implications for patient selection and monitoring therapy. $\left[{ }^{64} \mathrm{Cu}\right] \mathrm{WL} 12$ uptake in the tumors is highest at $60 \mathrm{~min}$ utes $(14.9 \% \mathrm{ID} / \mathrm{g})$ and reduced by 15 to $12.6 \% \mathrm{ID} / \mathrm{g}$ at 120 minutes (20). The image contrast is high at 120 minutes due to clearance from normal tissues. The fast clearance of the peptide suggests a washout from the tumor within 24 hours. Also, WL12 has lower binding affinity for PD-L1 than antibodies. Therefore, we do not expect $\left[{ }^{64} \mathrm{Cu}\right] \mathrm{WL} 12$ to interfere with therapeutic efficacy of antiPD-L1 antibodies, as it is given as a tracer dose (below $\mu \mathrm{g} / \mathrm{kg}$ ). Our $\left[{ }^{64} \mathrm{Cu}\right]$ WL12 PET measurements provide relative changes in target expression rather than true expression and such measurements acquired using PET are routinely used in the clinic (51). Other methods such as flow cytometry or autoradiography allow quantification of true expression; however, PET facilitates quantification of unoccupied-PD-L1 levels in real time and the measurements take all the PK/PD-induced changes in antibody concentration at the tumor into account that are difficult to capture using in vitro or ex vivo studies.

Until now, the tools to establish the relevance of dose to drug accumulation and target saturation, which is relevant to efficacy, have been unavailable for immune checkpoint therapeutics. Nearly
$25 \%$ of all immune checkpoint therapy clinical trials target PD-L1, and nearly $70 \%$ of involved patients do not respond to treatment (12). What is unknown is whether the drug fully engages the target in vivo successfully, or whether the tumor is immunologically resistant. Another question is to what extent does tumor microenvironment (stroma and extracellular matrix [ECM]) contribute to immunological resistance by hindering therapeutic accumulation in some lesions. Tumors display an abnormal microenvironment and ECM that are shaped by the tumor location, and influence drug accumulation. Analysis of tissue from multiple disease sites from a patient with melanoma showed an upregulation of genes that define the ECM in nonresponding lesions (52). The role of the ECM in tumor resistance to antibody transport has been demonstrated previously, but has not been established in the context of immune checkpoint therapeutics (53). We have used subcutaneous lung cancer xenografts, which do not fully capture the microenvironment of the orthotopic tumors and could potentially influence antibody accumulation. Nevertheless, our PET imaging approach will enable measurements of total PD-L1 and its saturation by the antibodies at every identifiable tumor while accounting for changes in ECM, facilitating therapy optimization across multiple tumor types.

Radiolabeled antibodies such as AtzMab, supported by peripheral pharmacodynamics assessments and PK/PD modeling, are routinely used to predict the antibody dosing levels required to achieve the desired PD-L1 occupancy at the tumor (29). Those measurements of radiolabeled-antibody concentrations in the plasma and tumor, as well as the subsequent occupancy predictions derived through mathematical modeling, are often specific to a given antibody, limiting generalization of such observations to other PD-L1 antibodies (54). The absence of an effective, widely applicable method that accounts for complex antibody kinetics and provides real-time PD-L1 saturation/occupancy data impedes optimized dose selection and therapeutic development. Our in silico, in vitro, and in vivo data using $\left[{ }^{64} \mathrm{Cu}\right] \mathrm{WL} 12$ PET demonstrate that PD-L1 saturation/occupancy can be noninvasively quantified at the tumor, a concept that can address this unmet need.

We provide a tool that can be used to assess antibody kinetics and target engagement potential for an ever-expanding array of PD-L1 therapeutic antibodies (32). Our data demonstrate that dynamic changes in PD-L1 expression in tumors, and PD-L1 saturation/occupancy by therapeutic antibodies, can be noninvasively measured independently of antibody selection. Imaging markers that directly reflect the levels and occupancy of PD-L1 have the potential to be more predictive, and form a bridge between dose, PD-L1 levels in the tumors, and therapeutic response. To the best of our knowledge our method provides the first noninvasive and translational tool to measure antibody target engagement at PD-L1, which is the necessary initial step toward finding safe, effective, and personalized doses for immunotherapy based on checkpoint inhibition. Importantly, this tool can be used to rapidly develop other tumor-targeted antibodies, significantly reducing time to approval and cost of development.

\section{Methods}

Study design. The goal of this study was to develop a noninvasive method to quantify the unoccupied fraction of PD-L1 and PD-L1 engagement by therapeutic antibodies. We performed a structural analysis 
of PD-L1, PD-1, and PD-L1 binding peptide $\left(\left[{ }^{64} \mathrm{Cu}\right] \mathrm{WL} 12\right)$ interactions and observed that $\left.{ }^{[64} \mathrm{Cu}\right] \mathrm{WL} 12$ could be used to evaluate the unoccupied fraction of PD-L1. We validated $\left[{ }^{64} \mathrm{Cu}\right]$ WL12 to quantify unoccupied-PD-L1 levels in vitro and in vivo using multiple cell lines and xenografts with graded levels of PD-L1 expression using 3 FDAapproved PD-L1 mAbs: AtzMab, AveMab, and DurMab. We then showed that $\left[{ }^{64} \mathrm{Cu}\right]$ WL12 can be used to evaluate the effect of dose of antibody and time on the unoccupied fraction of tumor PD-L1 following treatment, and used that data for the prediction of the dose of antibody needed to achieve therapeutically effective occupancy (defined as $>90 \%$ ) at the tumor by mathematical modeling.

Chemicals. WL12 was custom synthesized by CPC Scientific with greater than 95\% purity. 2,2',2"-(10-(2,6-Dioxotetrahydro$2 H$-pyran-3-yl)-1,4,7,10-tetraazacyclododecane-1,4,7-triyl)triacetic acid (DOTAGA anhydride) was purchased from CheMatech Macrocycle Design Technologies (catalog C109). All other chemicals were purchased from Sigma-Aldrich or Thermo Fisher Scientific. We synthesized WL12-DOTAGA as previously described by us $(20)$. [ $\left.{ }^{64} \mathrm{Cu}\right] \mathrm{CuCl}_{2}$ was purchased from the University of Wisconsin (Madison, Wisconsin, USA) and Washington University (St. Louis, Missouri, USA).

Cell culture reagents and antibodies. All cell culture reagents were purchased from Invitrogen. The anti-human PD-L1 mAb AtzMab and anti-CXCR4 mAb ulocuplumab (55) were generated as described previously (56), and AveMab and DurMab were purchased from the Johns Hopkins Hospital pharmacy.

Docking studies. The structure files for PD-L1 bound to PD-1 (57), AveMab (58), AtzMab (59), DurMab (59), BMS936559 (60), and KNO35 (61) were obtained from the Protein Data Bank (62), and compared to the predicted binding conformation of the cyclical peptide WL12 (20). The bound antibody and peptide structures were first aligned around the core of the PD-L1 interaction interface (residues 53-69), and the contact between the molecular surface of each antibody with PD-L1 was then identified using the Visual Molecular Dynamics software package (63).

Radiopharmaceutical preparation. ${ }^{64} \mathrm{CuCl}_{2}$ was buffered with 100 $\mathrm{mM}$ sodium acetate solution ( $\mathrm{pH} 5.5$ ) and converted to ${ }^{64} \mathrm{Cu}(\mathrm{OAc})_{2}$. For radiolabeling, approximately $20 \mu \mathrm{g}$ of WL12-DOTAGA peptide conjugate $(4.27 \mathrm{nmol})$, in $100 \mu \mathrm{l}$ sodium acetate, was mixed with $3-5 \mathrm{mCi}$ of ${ }^{64} \mathrm{Cu}(\mathrm{OAc})_{2}$ and incubated at $65^{\circ} \mathrm{C}$ for 30 minutes. The resulting radiotracer was purified on a C-18 column (Luna, $5 \mu \mathrm{m}, 10 \times 250 \mathrm{~mm}$; Phenomenex) using an elution gradient of $60 \%$ methanol ( $0.1 \%$ trifluoroacetic acid [TFA]) reaching $10 \%$ (with $\mathrm{H}_{2} \mathrm{O}, 0.1 \% \mathrm{TFA}$ ), over 30 minutes at a flow rate of $5 \mathrm{ml} / \mathrm{min}$. HPLC was performed on a Varian ProStar system equipped with a radioactive single-channel radiation detector (model 105S; Bioscan) and a Varian ProStar UV absorbance detector set to $280 \mathrm{~nm}$. [ ${ }^{64} \mathrm{Cu}$ ]WL12 was collected at 14.5 minutes, evaporated, formulated with saline containing 5\% DMSO and 1 drop of Tween 20, and used for in vitro and in vivo evaluation. $\left[{ }^{64} \mathrm{Cu}\right] \mathrm{WL} 12$ was obtained in $62.58 \% \pm 1.9 \%$ yield, with a specific activity of $1.9 \pm 0.11 \mathrm{mCi} / \mu \mathrm{g}$.

Fluorescent labeling of AtzMab, AveMab, and DurMab. To conjugate the fluorophore to the therapeutic mAbs, we used Cy5 NHS ester (excitation/emission of 650/669). Briefly, $1 \mathrm{mg}$ of mAb (6.5 nmol) in 400 $\mu \mathrm{l}$ of PBS was mixed with $4 \mu \mathrm{l}$ of DMSO containing $40 \mu \mathrm{g}$ of Cy5 NHS ester (65 nmol, GE, catalog 45-001-190). The reaction mixture was gently stirred for 2 hours at room temperature, followed by purification using Zeba spin desalting columns, 7K MWCO, $0.5 \mathrm{ml}$ (Thermo Fisher Scientific, catalog 89882) pre-equilibrated in PBS. Analysis of the Cy5-
mAbs, carried out using a Nanodrop 2000 UV-Vis spectrophotometer (Thermo Fisher Scientific), indicated a 1:2 mAb/Cy5 molar ratio.

MALDI-TOF analysis of antibodies. MALDI-TOF spectra of Cy5conjugated and parent mAbs (AtzMab, AveMab, and DurMab) were obtained on a Voyager DE-STR MALDI-TOF available at the Johns Hopkins University Mass Spectrometry core facility. Briefly, mAb samples were equilibrated in water with 0.1\% TFA using Amicon Ultra-15 centrifugal filter units (catalog UFC901008). Samples were mixed (1:2 dilution) with $10 \mathrm{mg} / \mathrm{ml}$ sinapinic acid (3,5-dimethoxy-4-hydroxycinnamic acid) matrix dissolved in $40 \%$ acetonitrile and $0.1 \%$ TFA. One microliter of sample was spotted in quadruplet on a MALDI plate (Applied Biosystems) and allowed to air dry, followed by spectra acquisition using optimized instrument settings. Data were analyzed using Applied Biosystems Data Explorer software version 4.8.

Cell culture. The A549, H226, HCC827, MDAMB231, and CHO cells were purchased from the American Type Culture Collection (ATCC). The CHO cells constitutively expressing PD-L1 (hPD-L1) were generated in our laboratory, and cultured as previously described (22). The SUM149 cells were obtained from Stephen Ethier (now at Medical University of South Carolina, Charleston, South Carolina, USA). All the cell lines were authenticated by STR profiling at the Johns Hopkins Genetic Resources Core Facility. The SUM149 cells were maintained in Ham's F-12 medium with 5\% FBS, 1\% penicillin/ streptomycin, $5 \mu \mathrm{g} / \mathrm{ml}$ insulin, and $0.5 \mu \mathrm{g} / \mathrm{ml}$ hydrocortisone. All other cell lines were cultured in ATCC-recommended media, in an incubator at $37^{\circ} \mathrm{C}$ in an atmosphere containing $5 \% \mathrm{CO}_{2}$.

Inducible PD-L1 expression vector. To generate a doxycyclineinducible PD-L1 (B7-H1, CD274) lentiviral expression vector, we Gateway cloned the full-length cDNA from pENTR223-CD274 (ORFeome Collaboration clone ccsbBroadEn_03086; DNASU Plasmid Repository clone HsCD00508899) into pINDUCER20, a gift from Stephen Elledge (Harvard Medical School, Boston, Massachusetts, USA) (Addgene plasmid 44012), by LR reaction $(26,64)$. The reaction mixture was transformed into Stbl3 cells and selected on LB agar plates supplemented with $100 \mu \mathrm{g} / \mathrm{ml}$ carbenicillin. The purified recombinant plasmid (pINDUCER20-CD274) was analyzed via BsrGI restriction enzyme digestion, and the sequence confirmed by Sanger sequencing.

Lentiviral production. The day before transfection, $3.4 \times 10^{6} \mathrm{HEK}-$ 293 T cells were plated in a $75-\mathrm{cm}^{2}$ flask. The next day, the cells were transfected with lentiviral plasmids by polyethylinimine transfection, in a 3:2:1 ratio of pINDUCER20-CD274/psPAX2/pMD2.G. The lentiviral packaging plasmids pMD2.G (Addgene plasmid 12259) and psPAX2 (Addgene plasmid 12260) were gifts from Didier Trono (EPFL, Lausanne, Switzerland). At 24 hours after transfection, the media were changed to complete media. Seventy-two hours after transfection, the lentivirus was harvested, filtered through a $0.45-\mu \mathrm{m}$ syringe filter, and stored at $-80^{\circ} \mathrm{C}$.

Lentiviral transduction. On day one, $1.5 \times 10^{5}$ A549 cells were plated in a $25-\mathrm{cm}^{2}$ flask. On day 2 , the cells were infected with $75 \mu \mathrm{l}$ of lentiviral supernatant in $5 \mathrm{ml}$ of complete media supplemented with $8 \mu \mathrm{g} / \mathrm{ml}$ polybrene. Twelve hours following lentiviral transduction, the media were removed and replaced with fresh complete media. To select for transduced cells, the media were replaced the following day with media containing G418 sulfate (Thermo Fisher Scientific, BP673) at a final concentration of $400 \mu \mathrm{g} / \mathrm{ml}$. The PD-L1 expression in stable clones was analyzed by flow cytometry. 
Detection of PD-L1 expression by flow cytometry and competitive inhibition assays. Cells were evaluated for PD-L1 surface expression by direct staining of $2 \times 10^{5}$ cells in $100 \mu \mathrm{l}$ PBS with Cy5-conjugated PD-L1 mAbs, for 30 minutes at $4^{\circ} \mathrm{C}$. Cells were then washed and analyzed for MFI by flow cytometry. Adherent cells were detached using enzyme-free cell dissociation buffer (Thermo Fisher Scientific).

Competitive inhibition assays were performed in $100 \mu$ l total volume in FACS buffer ( $1 \times$ PBS with 2 mM EDTA and 0.5\% FBS). The WL12 peptide was dissolved in DMSO to generate a $412 \mu \mathrm{M}$ stock to make $4 \times$ and $10 \times$ intermediate concentrations. WL12 was tested at 10 concentrations (0.1, 0.2, 0.3, 0.9, 2.7, 8.1, 15, 30, 60, and $100 \mathrm{nM})$. The WL12 stock $(10 \mu \mathrm{l})$ was added to $2 \times 10^{5} \mathrm{hPD}-\mathrm{L} 1$ or MDAMB231 cells in $80 \mu \mathrm{l}$ and incubated on ice for 30 minutes. Then, $10 \mu \mathrm{l}$ of Cy5-conjugated $\mathrm{mAb}$ was added to the cells, to reach a final concentration of $2 \mathrm{nM}$ for AtzMab, AveMab, and ulocuplumab, and $1 \mathrm{nM}$ for DurMab, and incubated for 30 minutes at $4^{\circ} \mathrm{C}$. Cells were then washed and analyzed for MFI by flow cytometry. The $\mathrm{IC}_{50}$ values were calculated based on normalized MFI values using GraphPad Prism 6 software. Data were acquired on an Accuri flow cytometer (Becton Dickinson) and at least 20,000 events were recorded.

In vitro binding assays with $\left[{ }^{64} \mathrm{Cu}\right] W L 12$. In vitro binding of $\left[{ }^{64} \mathrm{Cu}\right]$ WL12 to H226, HCC827, hPD-L1, CHO, MDAMB231, SUM149, A549, and A549-iPDL1 cells was determined by incubating $1 \times 10^{6}$ cells with approximately $0.1 \mu \mathrm{Ci}$ of $\left[{ }^{64} \mathrm{Cu}\right] \mathrm{WL} 12$ in the presence or absence of 60 $\mathrm{nM} \mathrm{mAb}$ for 30 minutes at $4^{\circ} \mathrm{C}$. After incubation, cells were washed 3 times with ice-cold PBS containing 0.1\% Tween 20, and counted on an automated gamma counter (1282 Compugamma CS, Pharmacia/ LKBNuclear, Inc.). To demonstrate PD-L1-specific binding of $\left[{ }^{64} \mathrm{Cu}\right]$ WL12, blocking was performed with $1 \mu \mathrm{M}$ nonradioactive WL12. All the cell radioactivity uptake studies were performed in quadruplicate for each cell line, and repeated 3 times.

In vivo studies. To establish xenografts, we used 5- to 6-week-old, male or female, nonobese, diabetic, severe-combined immunodeficient gamma (NSG) mice, obtained from the Johns Hopkins University Animal Resources Core.

Xenograft models. Mice were implanted with $\mathrm{H} 226\left(5 \times 10^{6}\right.$, s.c.), HCC827 $\left(5 \times 10^{6}\right.$, s.c. $)$, hPD-L1 $\left(10 \times 10^{6}\right.$, s.c. $)$, CHO $\left(10 \times 10^{6}\right.$, s.c. $)$, MDAMB231 $\left(2 \times 10^{6}\right.$, orthotopic), A549 $\left(5 \times 10^{6}\right.$, s.c.), or A549-iPDL (5 $\times 10^{6}$, s.c.) cells in their upper flanks. If 2 cell lines were used, cells were inoculated in the opposite flanks. For treatment, imaging, or biodistribution experiments, mice with tumor volumes of $100-300 \mathrm{~mm}^{3}$ were used.

To induce PD-L1 expression in A549-iPDL1 tumors, mice were given doxycycline ad libitum (10 $\mathrm{mg} / \mathrm{ml}$ doxycycline plus $1 \%$ sucrose $)$ in drinking water 72 hours prior to $\left[{ }^{64} \mathrm{Cu}\right] \mathrm{WL} 12$ injection.

For occupancy determination of AtzMab, AveMab, and DurMab at the tumor, xenograft-bearing mice $(n=9-12)$ were treated with a single intravenous bolus dose of either $20 \mathrm{mg} / \mathrm{kg}$ AtzMab, $10 \mathrm{mg} / \mathrm{kg}$ AveMab, or $10 \mathrm{mg} / \mathrm{kg}$ DurMab. Mice were used for imaging $(n=3-4)$ and biodistribution studies $(n=6-8) 24$ hours after mAb treatment.

For determining dose effect on the unoccupied fraction of PD-L1 in the tumor, MDAMB231 tumor-bearing mice were treated with a single intravenous bolus escalating dose of AtzMab, and 24 hours later mice were used for imaging $(n=3-4)$ and biodistribution $(n=6-8)$ studies. The PET imaging studies were performed in $0.06,0.6$, and $3.2 \mathrm{mg} /$ $\mathrm{kg}$ dose cohorts. To evaluate time effect on the unoccupied fraction of PD-L1 in the tumor after treatment, MDAMB231 tumor-bearing mice were treated with a single intravenous bolus dose of AtzMab $(0.06,1$, and $20 \mathrm{mg} / \mathrm{kg}$ ), and then imaging $(n=3)$ and biodistribution studies ( $n=5-8)$ were conducted at 24 and 120 hours after AtzMab treatment. The PET imaging studies were performed in $0.06 \mathrm{mg} / \mathrm{kg}$ dose cohorts.

PET-CT imaging of mouse xenografts. Mice treated for 24 hours with therapeutic $\mathrm{mAbs}$, or saline formulation as a control, were injected with $200 \mu \mathrm{Ci}$ of $\left[{ }^{64} \mathrm{Cu}\right] \mathrm{WL} 12$ in $200 \mu$ l of formulation intravenously, and PET images were acquired 2 hours after the injection of the radiotracer. For imaging, anesthesia was induced with $3 \%$ isoflurane, and maintained with $1 \%$ isoflurane. Images were acquired in 2 bed positions at $7 \mathrm{~min} /$ bed in an ARGUS small-animal PET/CT scanner (Sedecal). A CT scan (512 projections) was performed at the end of each PET scan for anatomical coregistration. The PET data were reconstructed using the 2-dimensional ordered subsets-expectation maximization algorithm (2D-OSEM), and corrected for dead time and radioactive decay. The \%ID per cc values were calculated based on a calibration factor obtained from a known radioactive quantity. Image fusion, visualization, and 3D rendering were accomplished using Amira 6.1 (FEI).

Ex vivo biodistribution. Mice harboring tumors and treated with therapeutic mAbs, or saline as a control $(n=5-8)$, were injected intravenously with $20 \mu \mathrm{Ci}$ of $\left[{ }^{64} \mathrm{Cu}\right] \mathrm{WL} 12$. At 2 hours after $\left[{ }^{64} \mathrm{Cu}\right] \mathrm{WL} 12$ injection, blood, tumors, and selected tissues (heart, lungs, liver, spleen, kidney, muscle) were harvested, weighed, and counted in an automated gamma counter (PerkinElmer, 2480 Wizard2 automatic gamma counter). Values for percentage of injected dose per gram of tissue (\%ID/g) were calculated based on signal decay correction, and normalization to external $\left[{ }^{64} \mathrm{Cu}\right]$ standards; measurements were made in triplicate. The biodistribution data shown are presented as mean \pm the standard error of the mean (SEM).

Relationship between PD-L1 mAb dose and PD-L1 engagement. To study the relationship between the AtzMab dose and unoccupied PD-L1 in the tumor, 13 dose levels were tested (0, 0.0009, 0.002, 0.006, $0.019,0.06,0.19,0.6,1.2,3.6,6,12$, and $24 \mathrm{mg} / \mathrm{kg}$ ) in MDAMB231 tumor-bearing mice. The numbers of mice tested were the following: 9 mice for anti-PD-L1 doses of $0 \mathrm{mg} / \mathrm{kg}$ and $1.2 \mathrm{mg} / \mathrm{kg}$ (pooled from 2 repeats), 5 mice for $0.0009 \mathrm{mg} / \mathrm{kg}$ to $0.6 \mathrm{mg} / \mathrm{kg}$, and 4 mice for 3.6 to $24 \mathrm{mg} / \mathrm{kg}$. Each mouse first received a single dose of AtzMab and 24 hours later was injected with $\left[{ }^{64} \mathrm{Cu}\right] \mathrm{WL} 12$. The radioactivity, measured 2 hours after the radiotracer injection, represented the unoccupied $\mathrm{PD}-\mathrm{L} 1$ remaining in the tumor site, and is expressed as \% ID $/ \mathrm{g}$.

An inhibitory sigmoidal $\mathrm{E}_{\max }$ model was used to describe the effect of AtzMab dose on the unoccupied PD-L1 measured at the tumor site. The decline in unoccupied PD-L1 in the presence of AtzMab was modeled as a proportional decrease from the baseline of unoccupied PD-L1 measured in the tumor in the absence of drug, as follows: Baseline unoccupied $P D-L 1_{1}$ is the baseline-measured unoccupied PD-L1 (in \%ID/g) in the absence of AtzMab $(0 \mathrm{mg} /$ $\mathrm{kg}$ dose); $I_{\max }$ is the maximum inhibitory effect, i.e., the maximum fractional decrease in unoccupied PD-L1 from Baseline unoccupied $P D-L 1$ $\left(0<I_{\max } \leq 1\right) ; I D_{50}$ is the AtzMab dose responsible for $50 \%$ of the maximum inhibitory effect $\left(I_{\max } / 2\right)$; and Hill is the Hill coefficient describing the slope or steepness of the relationship between the AtzMab dose and the unoccupied PD-L1.

The use of a proportional inhibitory $\mathrm{E}_{\max }$ model, to describe the relationship between AtzMab dose and unoccupied PD-L1 in the tumor, was considered more physiological than a conventional simple additive inhibitory $\mathrm{E}_{\max }$ model, where the inhibitory effect of a drug is constant and assumed independent of the extent of expression of the 
target or ligand at baseline. A naive-pooled approach was used to fit the data with the proportional inhibitory sigmoidal $\mathrm{E}_{\text {max }}$ model described above. All observations were treated as being measured from a single mouse, with repeated measurement at each dose level, because of the small range and variability in body weight of the studied mice, and that each mouse contributed only one unoccupied-PD-L1 measurement.

The statistical model used to describe the residual error variability in unoccupied-PD-L1 measurements (pharmacodynamic response) was an additive error model of the following form: Observed unoccupied $P D-L 1$ is the measured radioactivity or pharmacodynamic response; Predicted unoccupied $P D-L 1$ is the model-predicted pharmacodynamic response; and epsilon is the residual error term, assumed to follow a normal distribution with a mean zero and variance $\sigma^{2}$.

The developed model was used to determine $\mathrm{ID}_{50}$ of the AtzMab used in this study, and to derive the $\mathrm{ID}_{90}$ and $\mathrm{ID}_{96}$, responsible for $90 \%$ and $96 \%$ of $I_{\max }$, respectively. The expected approximate average plasma concentrations corresponding to each of the $\mathrm{ID}_{50}, \mathrm{ID}_{90}$, and $\mathrm{ID}_{96}$ doses were also tentatively derived by dividing each dose by the steady-state volume of distribution $\left(\mathrm{V}_{\mathrm{ss}}\right)$ of the anti-PD-L1 mAb in mice. Although the actual $V_{s s}$ of the studied AtzMab was unknown (no pharmacokinetic study was conducted), it was assumed to be close to the reported $\mathrm{V}_{\mathrm{ss}}$ of 42.6 to $57.5 \mathrm{ml} / \mathrm{kg}$, estimated in $\mathrm{BALB} / \mathrm{c}$ mice for the chimeric anti-PD-L1 antibody PRO304397 (mouse IgG2a antibody containing the binding region of atezolizumab).

Statistics. Statistical analyses were performed using GraphPad Prism 6 software. Unpaired 2-tailed Student's $t$ test, 1-way ANOVA, and 2-way ANOVA were utilized for column, multiple-column, and grouped analyses, respectively. Data represent mean \pm SEM. $P$ values $<$ 0.05 were considered statistically significant. AtzMab dose and PD-L1 engagement modeling was conducted using the nonlinear mixedeffects modeling program NONMEM (ICON Development Solutions).

Study approval. All mouse studies were conducted through Johns Hopkins University IACUC-approved protocols.

\section{Author contributions}

DK and AL designed the research studies, conducted radiochemistry and in vivo experiments, analyzed data, and assisted with the preparation of the manuscript. ED, MM, BW, SS, SC, WGL, PSS, and ET conducted in silico, in vitro, and ex vivo experiments, and acquired and analyzed data. VA, JTP, and CMR provided reagents. MGP, EMJ, EG, and JVSG provided technical and conceptual advice. SN conceived the idea, designed research studies, conducted experiments, analyzed data, prepared the manuscript, and supervised the study. All authors contributed to the writing and review of the manuscript.

\section{Acknowledgments}

We would like thank the University of Wisconsin and Washington University Cyclotron teams for ${ }^{64} \mathrm{CuCl}_{2}$ production. Funding for this study was provided by Allegheny Health Network-Johns Hopkins Cancer Research Fund (to SN), NIH 1R01CA236616 (to SN), and NIH P41EBO24495 (to MGP). Core resources (flow cytometry, histology, and imaging) were supported by NIH P30CA006973.

Address correspondence to: Sridhar Nimmagadda, Johns Hopkins Medical Institutions, 1550 Orleans Street, CRB II, Rm 491, Baltimore, Maryland 21287, USA. Phone: 410.502.6244; Email: snimmag1@jhmi.edu.
1. Arrowsmith J. Trial watch: Phase II failures: 2008-2010. Nat Rev Drug Discov. 2011;10(5):328-329.

2. Arrowsmith J, Miller P. Trial watch: phase II and phase III attrition rates 2011-2012. Nat Rev Drug Discov. 2013;12(8):569.

3. Morgan P, et al. Can the flow of medicines be improved? Fundamental pharmacokinetic and pharmacological principles toward improving Phase II survival. Drug Discov Today. 2012;17(9-10):419-424.

4. Jesudason CD, DuBois S, Johnson M, Barth VN, Need AB. In vivo receptor occupancy in rodents by LC-MS/MS. In: Sittampalam GS, et al, eds. Assay Guidance Manual. Bethesda,MD: Eli Lilly \& Company and the National Center for Advancing Translational Sciences; 2004.

5. Martinez Molina D, et al. Monitoring drug target engagement in cells and tissues using the cellular thermal shift assay. Science. 2013;341(6141):84-87.

6. Dubach JM, et al. Quantitating drug-target engagement in single cells in vitro and in vivo. Nat Chem Biol. 2017;13(2):168-173.

7. Burr ML, et al. CMTM6 maintains the expression of PD-L1 and regulates anti-tumour immunity. Nature. 2017;549(7670):101-105.

8. Mezzadra R, et al. Identification of CMTM6 and CMTM4 as PD-L1 protein regulators. Nature. 2017;549(7670):106-110.

9. Coelho MA, et al. Oncogenic RAS signaling promotes tumor immunoresistance by stabilizing
PD-L1 mRNA. Immunity. 2017; 47(6):1083-1099.e6.

10. Willmann JK, van Bruggen N, Dinkelborg LM, Gambhir SS. Molecular imaging in drug development. Nat Rev Drug Discov. 2008;7(7):591-607.

11. Rathkopf DE, et al. Phase I study of ARN-509, a novel antiandrogen, in the treatment of castration-resistant prostate cancer. J Clin Oncol. 2013;31(28):3525-3530.

12. Nishino M, Ramaiya NH, Hatabu H, Hodi FS. Monitoring immune-checkpoint blockade: response evaluation and biomarker development. Nat Rev Clin Oncol. 2017;14(11):655-668.

13. Yarchoan M, Hopkins A, Jaffee EM. Tumor mutational burden and response rate to PD-1 inhibition. N Engl J Med. 2017;377(25):2500-2501.

14. Sharma P, Hu-Lieskovan S, Wargo JA, Ribas A. Primary, adaptive, and acquired resistance to cancer immunotherapy. Cell. 2017;168(4):707-723.

15. Syn NL, Teng MWL, Mok TSK, Soo RA. De-novo and acquired resistance to immune checkpoint targeting. Lancet Oncol. 2017;18(12):e731-e741.

16. Hegde PS, Karanikas V, Evers S. The where, the when, and the how of immune monitoring for cancer immunotherapies in the era of checkpoint inhibition. Clin Cancer Res. 2016;22(8):1865-1874

17. Agoram BM. Use of pharmacokinetic/pharmacodynamic modelling for starting dose selection in first-in-human trials of high-risk biologics. $\mathrm{Br} \mathrm{J}$ Clin Pharmacol. 2009;67(2):153-160.

18. Brahmer JR, et al. Safety and activity of anti-
PD-L1 antibody in patients with advanced cancer. N Engl JMed. 2012;366(26):2455-2465.

19. Lamberts LE, et al. Antibody positron emission tomography imaging in anticancer drug development. J Clin Oncol. 2015;33(13):1491-1504.

20. Chatterjee S, et al. Rapid PD-L1 detection in tumors with PET using a highly specific peptide. Biochem Biophys Res Commun. 2017;483(1):258-263.

21. Magiera-Mularz K, et al. Bioactive macrocyclic inhibitors of the PD-1/PD-L1 immune checkpoint. Angew Chem Int Ed Engl. 2017;56(44):13732-13735.

22. Chatterjee $S$, et al. A humanized antibody for imaging immune checkpoint ligand PD-L1 expression in tumors. Oncotarget. 2016;7(9):10215-10227.

23. Mansfield AS, Dong H. Implications of programmed cell death 1 ligand 1 heterogeneity in the selection of patients with non-small cell lung cancer to receive immunotherapy. Clin Pharmacol Ther. 2016;100(3):220-222.

24. Taube JM, et al. Differential expression of immune-regulatory genes associated with PD-L1 display in melanoma: Implications for PD-1 pathway blockade. Clin Cancer Res. 2015;21(17):3969-3976.

25. Taube JM, et al. Colocalization of inflammatory response with B7-H1 expression in human melanocytic lesions supports an adaptive resistance mechanism of immune escape. Sci Transl Med. 2012;4(127):127ra37. 
26. Meerbrey KL, et al. The pINDUCER lentiviral toolkit for inducible RNA interference in vitro and in vivo. Proc Natl Acad Sci U S A 2011;108(9):3665-3670.

27. Heskamp S, et al. Noninvasive imaging of tumor PD-L1 expression using radiolabeled anti-PD-L1 antibodies. Cancer Res. 2015;75(14):2928-2936.

28. Maute RL, et al. Engineering high-affinity PD-1 variants for optimized immunotherapy and immuno-PET imaging. Proc Natl Acad Sci U S A 2015;112(47):E6506-E6514.

29. Deng R, et al. Preclinical pharmacokinetics, pharmacodynamics, tissue distribution, and tumor penetration of anti-PD-L1 monoclonal antibody, an immune checkpoint inhibitor. MAbs. 2016;8(3):593-603.

30. Hettich M, Braun F, Bartholomä MD, Schirmbeck R, Niedermann G. High-resolution PET imaging with therapeutic antibody-based PD-1/PD-L1 checkpoint tracers. Theranostics. 2016;6(10):1629-1640.

31. Josefsson A, et al. Imaging, biodistribution, and dosimetry of radionuclide-labeled PD-L1 antibody in an immunocompetent mouse model of breast cancer. Cancer Res. 2016;76(2):472-479.

32. Tang J, Shalabi A, Hubbard-Lucey VM. Comprehensive analysis of the clinical immuno-oncology landscape. Ann Oncol. 2018;29(1):84-91.

33. Tan S, et al. Distinct PD-L1 binding characteristics of therapeutic monoclonal antibody durvalumab. Protein Cell. 2018;9(1):135-139.

34. Herbst RS, et al. Predictive correlates of response to the anti-PD-L1 antibody MPDL3280A in cancer patients. Nature. 2014;515(7528):563-567.

35. Baxter LT, Jain RK. Transport of fluid and macromolecules in tumors. I. Role of interstitial pressure and convection. Microvasc Res. 1989;37(1):77-104.

36. Baxter LT, Jain RK. Transport of fluid and macromolecules in tumors. II. Role of heterogeneous perfusion and lymphatics. Microvasc Res. 1990;40(2):246-263.

37. Shah DK, Betts AM. Antibody biodistribution coefficients: inferring tissue concentrations of monoclonal antibodies based on the plasma concentrations in several preclinical species and human. MAbs. 2013;5(2):297-305.

38. Tabrizi MA, Tseng CM, Roskos LK. Elimination mechanisms of therapeutic monoclonal antibod- ies. Drug Discov Today. 2006;11(1-2):81-88.

39. Stroh M, et al. Clinical pharmacokinetics and pharmacodynamics of atezolizumab in metastatic urothelial carcinoma. Clin Pharmacol Ther. 2017;102(2):305-312.

40. Lindauer A, et al. Translational pharmacokinetic/ pharmacodynamic modeling of tumor growth inhibition supports dose-range selection of the anti-PD-1 antibody pembrolizumab. CPT Pharmacometrics Syst Pharmacol. 2017;6(1):11-20.

41. Lee CM, Farde L. Using positron emission tomography to facilitate CNS drug development. Trends Pharmacol Sci. 2006;27(6):310-316.

42. Sheng J, et al. Clinical Pharmacology considerations for the development of immune checkpoint inhibitors. JClin Pharmacol. 2017;57(Suppl 10):S26-S42.

43. Biotech $\mathrm{PhD}$. The strange tale of pidilizumab. The Science of Biotech. https://www.scienceofbiotech. com/strange-tale-pidilizumab/. Published March 10, 2017. Accessed November 27, 2018.

44. Roach C, et al. Development of a companion diagnostic PD-L1 immunohistochemistry assay for pembrolizumab therapy in non-small-cell lung cancer. Appl Immunohistochem Mol Morphol. 2016;24(6):392-397.

45. Meng X, Huang Z, Teng F, Xing L, Yu J. Predictive biomarkers in PD-1/PD-L1 checkpoint blockade immunotherapy. Cancer Treat Rev. 2015;41(10):868-876.

46. Ilie M, et al. Comparative study of the PD-L1 status between surgically resected specimens and matched biopsies of NSCLC patients reveal major discordances: a potential issue for anti-PD-L1 therapeutic strategies. Ann Oncol. 2016;27(1):147-153

47. McLaughlin J, et al. Quantitative assessment of the heterogeneity of PD-L1 expression in non-small-cell lung cancer. JAMA Oncol. 2016;2(1):46-54.

48. Nolan E, et al. Combined immune checkpoint blockade as a therapeutic strategy for BRCA1-mutated breast cancer. Sci Transl Med. 2017;9(393): eaal4922.

49. Gniadek TJ, Li QK, Tully E, Chatterjee S, Nimmagadda S, Gabrielson E. Heterogeneous expression of PD-L1 in pulmonary squamous cell carcinoma and adenocarcinoma: implications for assessment by small biopsy. Mod Pathol.
2017;30(4):530-538.

50. Gibney GT, Weiner LM, Atkins MB. Predictive biomarkers for checkpoint inhibitor-based immunotherapy. Lancet Oncol. 2016;17(12):e542-e551.

51. Weissleder R, Schwaiger MC, Gambhir SS, Hricak H. Imaging approaches to optimize molecular therapies. Sci Transl Med. 2016;8(355):355ps16

52. Ascierto ML, et al. Transcriptional mechanisms of resistance to anti-PD-1 therapy. Clin Cancer Res. 2017;23(12):3168-3180.

53. Netti PA, Berk DA, Swartz MA, Grodzinsky AJ, Jain RK. Role of extracellular matrix assembly in interstitial transport in solid tumors. Cancer Res. 2000;60(9):2497-2503.

54. Kamath AV. Translational pharmacokinetics and pharmacodynamics of monoclonal antibodies. Drug Discov Today Technol. 2016;21-22:75-83.

55. Azad BB, et al. A fully human CXCR4 antibody demonstrates diagnostic utility and therapeutic efficacy in solid tumor xenografts. Oncotarget. 2016;7(11):12344-12358.

56. Powles T, et al. MPDL3280A (anti-PD-L1) treatment leads to clinical activity in metastatic bladder cancer. Nature. 2014;515(7528):558-562.

57. Zak KM, et al. Structure of the complex of human programmed death 1, PD-1, and its ligand PD-L1. Structure. 2015;23(12):2341-2348.

58. Liu K, et al. Structural basis of anti-PD-L1 monoclonal antibody avelumab for tumor therapy. Cell Res. 2017;27(1):151-153.

59. Lee HT, et al. Molecular mechanism of PD-1/ PD-L1 blockade via anti-PD-L1 antibodies atezolizumab and durvalumab. Sci Rep 2017;7(1):5532.

60. Lee JY, et al. Structural basis of checkpoint blockade by monoclonal antibodies in cancer immunotherapy. Nat Commun. 2016;7:13354.

61. Zhang F, et al. Structural basis of a novel PD-L1 nanobody for immune checkpoint blockade. Cell Discov. 2017;3:17004

62. Berman HM, et al. The Protein Data Bank. Nucleic Acids Res. 2000;28(1):235-242.

63. Humphrey W, Dalke A, Schulten K. VMD: visual molecular dynamics. JMol Graph. 1996;14(1):33-38, 27-28.

64. Yang X, et al. A public genome-scale lentiviral expression library of human ORFs. Nat Methods. 2011;8(8):659-661. 